\title{
Beiträge zur Theorie des Mikroskops und der mikroskopischen Wahrnehmung.
}

I. Die Construction von Mikroskopen auf Grund der Theorie. II. Die dioptrischen Bedingungen der Leistung des Milkroskops. III. Die physikalischen Bedingungen für die Abbildung feiner Structuren. IV. Das optische Vermögen des Milkroskops.

Von

\section{Dr. Wobe,}

ao. Professor in Jena.

\section{Die Construction von Mikroskopen auf Grund der Theorie.}

1. In den Handbüchern der Mikrographie findet man gelegentlich die Thatsache berührt, dass die Construction der Miksroskope und ihre fortschreitende Verbesserung bisher fast ausschliesslich Sache der Empirie, geschickten und ausdauernden Probirens von Seiten erfahrener Praktiker, geblieben ist. Hin und wieder wird. auch wohl die Frage aufgeworfen: warum die Theorie, nach welcher man von der Wirkungsweise des fertigen Mikroskops genügend Rechenschaft geben kann, nicht zugleich die Grundlage für seine Herstellung geworden sei, warum man also nicht auch diese Art von optischen Instrumenten nach theoretisch entwickelten Rechnungsvorschriften construire, wie solches seit Fraunhofer mit dem Fernrohr und in neuerer Zeit mit den optischen Theilen der photographischen Camera so exfolgreich geschieht. Der Grund für das Fortbestehen des empirischen Verfalrens wird allgemein in technischen Schwierigkeiten gesucht - in der vermeintlichen Unmöglichkeit, bei der Ausführung der Mikroskopobjective vorgeschriebene 
Maasse für die einzelnen Bestandtheile der Construction in der erforderlichen Genauigkeit einzuhalten. Auf den ersten Blick erscheint diese Erklärung in der That als durchaus plausibel; denn die Kleinheit der Dimensionen, welche zumal bei den stärkeren Objectiven unvermeidlich ist, lässt die Schwierigkeiten ihrer Herstellung nach exacten Maassen als ausserordentlich gross ansehen. Nichtsdestoweniger hat sich mir dieses Bedenken als unzutreffend herausgestellt, nachdem ich nähere Kenntniss erlangt hatte über die Einrichtungen und die technischen Verfahrungsweisen, die in einer wohlgeleiteten optischen Werkstatt bei der Construction der Mikroskope in Anwendung kommen. Die aufmerksame Berücksichtigung der wissenschaftlichen und technischen Hilfsmittel, die der praktischen Optik zu Gebote stehen, und die Abwägung der verschiedenartigen Schwierigkeiten ain Leitfaden einer theoretischen Discussion der einschlagenden Bedingungen führte vielmehr zu der durch den schliesslichen Erfolg bewährten Ueberzeugung: dass für den dermaligen Stand der optischen Technik die Ausfuhrung von Linsen und Linsensystemen nach vorgeschriebenen Maassen aller Elemente, in einer die richtige Wirkung verbürgenden Genauigkeit, nicht schwieriger, sondern eher leichter sei als die Erfüllung der anderweitigen Ansprüche, welche auf alle Fälle gestellt werden müssen, wenn die betreffenden Dinge überhaupt brauchbar sein sollen; und dass es mithin nur darauf ankommen werde, alle Momente der optischen Wirkung richtig in Rechnung zu stellen, um des Erfolges einer theoretischen Construction bei der technischen Ausführung sicher zu sein. Auf diese Ansicht hin habe ich denn in Verbindung mit Herrn C. Zeiss in Jena einen ernsthaften Versuch unternommen, der Construction der Mikroskope und ihrer weitern Vervollkommnung eine ebenso sichere theoretische Grundlage zu geben, wie die Herstellung astronomischer Fernrohre schon durch Fraunhofer gewonnen hat. Dank der Bereitwilligkeit, mit welcher Herx Z e is s mir hierbei entgegengekommen ist, indem er mir mehrere Jahre hindurch die ausgezeichneten Hilfsmittel und die tüchtigen Arbeitskräfte seiner Werkstatt zur Verfügung stellte, und Dank dem Eifer, mit welchem der kunstfertige Werkführer dieser Werkstatt und seine geschickten Gehilfen den einschlagenden Arbeiten sich unterzogen haben, ist dieser Versuch nach längeren Bemühungen zum Ziele gelangt. Seit einiger Zeit werden in der genannten Werkstatt Mikroskopsysteme, die einigermassen auf der Höhe der dermaligen Lei- 
stungen stehen dürften, vom schwächsten bis zum stärksten, durchaus nach theoretischen Vorschriften ausgeführt.

Die betreffenden Constructionen sind dabei, auf Grund genauer Untersuchung der zu verwendenden Materialien, bis in die letzten Einzelheiten - jede Krümmung, jede Dicke, jede Linsenöffnung durch Rechnung festgestellt, so dass alles Tatonnement ausgeschlossen bleibt. Von jedem zu verarbeitenden Glasstück werden zuvor die optischen Constanten an einem Probeprisma mittelst des Spectrometers gemessen, um Abweichungen des Materials durch geeignete Veränderung der Construction unschïdlich zu machen. Die einzelnen Bestandtheile werden möglichst genau nach den vorgeschriebenen Maassen ausgeführt und zusammengesetzt, und nur bei den stärkeren Objectiven wird ein Element der Construction (eine Linsendistanz) bis zuletzt variabel gelassen, um mittelst desselben die unvermeidlichen kleinen Abweichungen der Arbeit wieder ausgleichen zu kömnen. - Es zeigt sich dabei, dass eine hinreichend gründliche Theorie in Verbindung mit einer rationellen Technils, die alle Hilfsmittel benutzt, welche die Physik der praktischen Optik bietet, auch bei der Construction der Milrroskope die empirischen Verfahrungsweisen mit Erfolg ersetzen kann.

2. Im Laufe der Arbeiten, welche zu diesem Resultat geführt haben, hat sich nun herausgestellt, dass die bisherige Theorie des Milkroskops in wesentlichen Stücken sehr unvollständig ist. Die Art zunächst, wie die Bedingungen einer vollkommenen Abbildung und darauf hin die Ursachen der Unvollkommenheit discutirt werden, zeigte sich der wirklichen Sachlage, wie sie beim Mikroskop besteht, nicht entfernt gewachsen. Der Umstand, dass hier eine Grösse des Oeffnungswinkels in Frage kommt, wie sie bei keinem andern optischen Instrument wiederkehrt, macht namentlich die angenommenen Begriffe der Aberrationen durchaus unbrauchbar schon für jede einigermassen erschöpfende und zutreffende Beurtheilung gegebener fertiger Mikroskope, geschweige denn für eine exacte Vorausbestimmung der Wirkungen einer erst auszuführenden Construction. Um für einen derartigen Versuch den erforderlichen Anhalt zu gewinnen, musste die theoretische Analyse der Wirkung eines Linsensystems von grossem Oeffnungswinkel auf eine viel breitere mathematische Grundlage gebaut und viel weiter in's Einzelne geführt werden, als bisher geschehen ist; wobei sich denn ergeben hat, dass die richtige Functionirung eines den heutigen Ansprüchen ge- 
nügenden Mikroskopsystems von einer unerwartet grossen Anzahl selbstständiger Bedingungen abhängt, deren sachgemässe Würdigung nicht möglich ist ohne die Einführung mancher neuer Gesichtspunkte in die allgemeine Theorie des Mikroskops.

Die Ergänzung der 'Theorie nach dieser Seite hin war in der Hauptsache eine rein mathematische Aufgabe, welche mit den feststehenden Grundsätzen der Dioptrik vollständig zu erledigen war. Erfahrung und Experiment kamen dabei nur in soweit in Frage, als es sich darum Handelte, die Exscheinungsform der einzelnen theoretisch nachweisbaren Fehlerquellen am fertigen Mikroskop kennen zu lernen und ihre sehr ungleiche Bedeutung für den pralstischen Gebrauch des Instruments richtig in Anschlag zu bringen. Dagegen stellte sich in. der bisherigen Kenntniss noch eine Lücke anderer Art heraus, welche nur durch erweiterte Erfahrung ausgefüllt werden konnte. Sie ist gekennzeichnet durch die unsichern, zum Theil einander widersprechenden Ansichten, welche über die Bedeutung: des Oeffnungswinkels der Objective und die sogen. Functionen des optischen Vermögens, Definition und Auflösung, aufgestellt werden. Die Unsicherheit in diesem Punkte $z a$ heben und eine deutliche Einsicht in die hier eingreifenden Bedingungen zu gewinnen, war die conditio sine qua non für jeden erfolgreichen Versuch in der angegebenen Richtung. Denn die Anforderung, welche an die Grösse des Oeffnungswinkels gestellt wird, giebt erst Maass und Richtschnur für die ganze Aufgabe. Alle Verhältnisse der Construction werden durchaus andere, je nachdem für ein Objectiv 40 oder 90 oder $150 \mathrm{Grad}$ als Oeffnungswinkel rorgeschrieben wird. Welche Ansprüche aber in dieser Hinsicht rationell seien, blieb völlig zweifelhaft, so lange sich nicht die genaueste Rechenschaft von der wirklichen Bedeutung dieses Factors geben liess.

3. Die Untersuchungen, welche ich ausgeführt habe, um diese Frage selbstständig zur Entscheidung zu bringen, haben das Ergebniss geliefert, dass ein wesentliches Moment in der optischen Function des Mikroskops bisher gänzlich übersehen worden ist. Bei der Erklärung und Deutung der Wirkungen dieses Instruments hat man es nämlich als eine selbstverständliche Sache angesehen, dass die Abbildung der mikroskopischen Objecte in allen Stücken nach denselben dioptrischen Gesetzen erfolge, nach denen mikroskopische Bilder im Fernrohr oder auf der Platte einer Camera erzeugt werden; and man hat darauf hin stillschweigend vorausgesetzt, dass 
alle optischen Functionen beim Mikroskop grade so wie bei diesen andern Apparaten durch die geometrisch definirbaren Verhältnisse des Strahlengangs bestimmt seien. Eine strengere Kritik der bekannten Erfahrungen, auf welche die traditionelle Unterscheidung von Definitions- und Aufösungs-Vermögen sich stützt, hat diese scheinbar so natürliche Annalime als unzulässig herausgestellt. Es hat sich gezeigt, dass sie zwar für gewisse, speciell nachweisbare Fälle giltig bleibt, dass aber im Allgemeinen, und zumal bei solchen Objecten, an denen das Mikroskop seine höchste Leistungsfähigkeit bewähren soll, die Erzengung der mikroskopischen Bilder an einen eigenthümlichen, bisher nicht beachteten physikalischen Process geknüpft ist, der in den Objecten selbst seinen Sitz hat, unabhängig von der Einrichtung der Mikroskope eintritt, für dessen Effecte aber diese letztern mittelbar maassgebend wird.

Die Consequenzen dieser Thatsachen reichen an die wichtigsten Probleme der Mikrographie heran. Sie führten zum Nachweis einer ganz specifischen Function des Oeffnungswinlrels und, in Anschluss hieran, auf deutliche und sichere Begriffe über das sogen. optische Vermögen des Mikroskops in seinen beiden Factoren, aus welchen Begriffen sich alle Bedingungen, von denen seine Leistung abhängt, genau feststellen lassen. Hieraus folgen zunächst bestimmte praktische Maximen für die rationelle Construction des Mikroskops, sowie Winke für eine sachgemässe Prüfung gegebener Instrumente. Andrerseits aber führte der Ausbau der so gewonnenen Grundlage durch Experiment und Theorie zu einigen die mikroskopische Wahrnehmung im Allgemeinen betreffenden Schlussfolgerungen. Nicht nur lässt sich eine Grenze der Kleinheit bestimmen, bei der alle Beobachtung mikroskopischer Structuren eine Schranke finden muss, sondern es tritt auch ein allgemein eingreifendes Moment zu Tage, welches beim wissenschaftlichen Gebrauch des Mikroskops nicht wird ausser Acht bleiben dürfen; indem sich zeigt, dass die bisher unangefochten gebliebene Grundlage für die Deutung mikroskopischer Wahrnehmungen - dass nämlich ein fehlerfreies mikroskopisches Bild in allen Fällen die wirlkliche Beschaffenheit des Objects darstelle - für eine ganze Classe von Beobachtungen durchaus nicht zu Recht besteht.

Die hier in ihren Hauptrichtungen bezeichneten theoretischen und experimentellen Studien waren zwar, ihrer Veranlassung nach, zunächst auf den praktisehen Zweck gerichtet, einen sicheren Leit- 
faden für die richtige Formulirung der Ansprüche bei der Berechnung von Linsensystemen zu gewinnen; sie haben sich aber von selbst zu einer vollständigen Theorie des Mikroskops abgerundet, welche so ziemlich in alle Capitel der mikrographischen Doctrin eingreift und dieser ausserdem einige neue Capitel hinzufügt. Dieser Theorie ist die enge Verbindung mit der Technik der Mikroskopverfertigung in zwiefacher Art zu Statten gekommen. Einestheils haben die strengen Anforderungen, welche der praktische Zweck der Arbeit stellte, Nachforschungen nöthig gemacht, zu welchen die Mikrographen schwerlich Anlass gefunden haben würden; andrerseits aber hat die wirkliche Ausführung von Mikroskopen nach den Grundsätzen der in Rede stehenden Theorie für alle wesentlichen Bestandtheile derselben die empfindlichste Probe herbeigefuhrt, welcher theoretische Ansichten auf diesem Felde unterworfen werden können.

Die detaillrte Mittheilung dieser Studien über die Theorie des Mikroskops und der mikroskopischen Wahrnehmung wird demnächst in einem ausführlichen Aufsatze im VIII. Bande der Jen a ischen Zeitschrift für Medicin und Naturwissenschaft erfolgen. Da ich jedoch annehme, dass unter den praktischen Mikroskopikern Manchem eine gedrängte Uebersicht über die Ergebnisse der Unter suchung willkommen sein wird, so erlaube ich mir, den Lesern dieses Archivs im Folgenden eine kurze Zusammenstellung der hauptsächlichsten Resultate meiner Arbeit rorzulegen. Ich folge dabei den beiden oben hervorgehobenen Richtungen der Untersuchung, indem ich zuerst Dasjenige anführe, was auf den rein dioptrischen Theil der Theorie abzielt und sodann übergehe zur Betrachtung der angedeuteten neuen Factoren, welche in die Functionen des Mikroskops eingreifen; wobei ich indess bemerken will, dass die folgenden Darlegungen an keiner Stelle den Anspruch machen, die vollständige Entwickelung und Begründung der aufzustellenden Sätze, wie sie der ausführliche Bericht liefern wird, reproduciren zu wollen.

\section{Die dioptrischen Bedingungen der Leistung des Mikroskops.}

4. Bei der Erklärung des Strahlenganges, der im Mikroskop die Abbildung der Objecte vermittelt, pflegt man zum Ausgangspunkt aller speciellen Untersuchungen das bekannte typische Schema des zusámmengesetzten Mikroskops zu wählen, welchem zufolge das Objectiv ein umgekehrtes Bild des Objects erzeugt und das Ocular, 
als Lupe wirkend, dieses vergrössert in die Weite des deutlichen Sehens rückt. Auf die solchem Schema entsprechende Zerlegung des Vorgangs gründet man zugleich die Discussion der näheren Bedingungen, von denen die optische Leistung nach Quantität und Qualität abhängt. - Zu einer allgemeinen Uebersicht über die Wirkungsart des Mikroskops reicht diese Betrachtungsweise allerdings aus. Soll jedoch die dioptrische Analyse des Abbildungsvorgangs die Grundlage gewähren für eine genauere Feststellung der verschiedenen Factoren, welche in ihm maassgebend sind, so bedarf dieselbe nach zwei Seiten hin einer wesentlichen Erweiterung.

Erstens muss der Strahlengang im Mikroskop unter einem allgemeineren Gesichtspunkt angesehen werden. Die nämlichen Strahlen, welche als homofocale Büschel von den einzelnen Objectpunkten aus im Mikroskop verlaufen, lassen sich auch zusammenfassen zu homofocalen Büscheln, die von den verschiedenen Punkten einer vor. (unter) dem Mikroskop im äussern Raume liegenden Fläche ausgehen. Diese Fläche ist im Allgemeinen die - dioptrisch nach aussen projicirt gedachte Oeffnung des Objectivs und enthält im Besondern die zur Beleuchtung dienende Lichtquelle (z. B. die Fläche des Beleuchtungsspiegels) als Theil in sich. Neben denjenigen Bildern, welche die Bestandtheile des optischen Systems succes sive vom Object entwerfen, den Objectbildern, entsteht daher eine Reihe von zugeordneten Oeff $n$ ung s bildern, welche sämmtlich die nach aussen projicirte Fläche der freien Oeffnung abbilden. Das letzte von diesen, das dem schliesslichen virtuellen Bilde des Objects zugeordnete, erscheint im sogen. Augenpunkt oberhalb des Oculars und kann daselbst mittelst einer Lupe näher beobachtet werden; das erste, vom Objectiv allein erzeugte, liegt in oder nahe der obern Focalebene des Objectivs und bietet sich dem freien Auge beim Hereinsehen in den offenen Tubus dar. - Beide Reihen von Bildern sind durch allgemeine Relationen untereinander verknüpft, deren Nachweis den Schlüssel für zahlreiche sonst schwer zugängliche Fragen abgiebt. Alle Merkmale der Objectbilder hängen mit gewissen andern Merkmalen der Oeffnungsbilder zusammen, und umgekehrt; und namentlich enthalten die letzteren alle Bestimmungsstücke für die Beg r enzung der Strahlenbüschel, welche die Erzeugang der ersteren vermitteln. - Darauf hin ergeben sich $\mathfrak{u}$. A. auf rein theoretischem Wege allgemein giltige Sätze über die sogen. Tiefenperspective des Mikroskops, über den Einfluss, den die Beugung des Lichts in der 
freien Oeffnung des Objectivs auf das mikroskopische Bild ausübt, vor Allem aber über die Bedingungen der Lichtstärke und über die Wirkung verschiedener Beleuchtungsweisen und verschiedener Beleuchtungsapparate. Andrerseits aber liefert die wirkliche Beobachtung der Oeffnungsbilder unter Benutzung geeigneter Vergrösserungsvorrichtungen ein neues Hilfsmittel zum Studium der Objecte selbst. In ihnen erscheinen nämlich die $\mathrm{S} p$ ur en der sämmtlichen Lichtstrahlen, die in irgend welchen Richtungen vom object aus in das Mikroskop gelangt sind; die erhellten Theile eines der Oeffnungsbilder, z. B. des ersten, in der obern Focalebene des Objectivs gelegenen, markiren die verschiedenen Strahlenbüschel, welche vom Präparat ausgehen und dessen Abbildung vermitteln. Daher müssen alle Einwirkungen, welche dieses Präparat auf die hindurchtretenden Lichtstrahlen ausübt, im Besondern alle Ablenliungen, als Modificationen in jenen Oeffnungsbildern direct erkennbar sein; was im Folgenden weitere Ausführung und mehrfache Anwendung finden wird.

Die Grundlage für die exacte Entwickelung der mannigfachen Folgerungen, zu denen die Betrachtung der erwähnten Oeffnungsbilder führt, bildet ein für die ganze Theorie des Mikroskops sehr fruchtbarer Satz, der sich allgemein erweisen lässt. Er lautet: Wenn ein optisches. System für einen seiner Brennpunkte vollkommen aplanatisch ist, so trifft jeder.von diesem Brennpunkte ausgehende strahl eine durch den andern Brennpunkt gelegte Ebene in einem $\mathrm{Ab}$ stande yon der Axe, dessen lineare Grösse gleich ist dem Product aus der Aequivalentbrennweite des Systems mit dem Sinus des Winkels, welchen der betreffende Strahl mit der Axe bildet. - Da die genannte Bedingung bei einem correcten Mikroskop sowohl für das Objectiv allein wie auch für das Mikroskop im Ganzen erfüllt sein muss, so stellt dieses Theorem einen quantitativen Zusammenhang, her zwischen dem Oeffnungs winkel des Mikroskops einerseits und den linearen Durchmessern der Oeffnungsbilder über dem Objectiv und über dem Ocular. Er macht es aber auch möglich, aus dem mikrom metrisch gemessenen Ort, den die Spur eines Strahles in der obern Focalebene des Objectivs einnimmt, die Richtung zu bestimmen, in welcher derselbe vor dem Eintritt in das Mikroskop verläuft. . Das Oeffnungsbild über dem Objectiv kann daher, wenn man es mit einem passenden Mikrometer-Ocular beobachtet, zur Messung der 
Ablenkungen benutzt werden, welche die Lichtstrahlen im Präparat erleiden. - Vergl. (15) u. f.

5. Zweitens bedarf es einer tiefer greifenden Charakteristik für die wesentlichen optischen Functionen, welche bei der Abbildung eines Objects unter beliebig gros s e m Bildwinkel und mit Strahlenkegeln von beliebig grossem Oeffnungswinkel den Abbildungsvorgang unterscheiden von der Abstraction, in der die Gaussische Theorie die Wirkungen eines Linsensystems betrachtet. Diese Charakteristik ergiebt sich durch die Aufstellung allgemeingiltiger Begriffe über die Focalwirlsung und die Flächenausbreitung in einem optischen System, durch welche zwei der Idee nach selbstständige und in ihren specifischen Leistungen auch thatsächlich trennbare Grundfactoren des Abbildungsvorgangs bezeichnet werden. Abgeschen davon, dass erst auf eine solche Unterscheidung hin eine erschöpfende Analyse der Vollkommenheitsbedingungen und der $\mathrm{Ab}$ bildungsfehler möglich wird, bietet dieselbe das einzige Hilfsmittel, um den Antheil der einzelnen Bestandtheile eines zusammengesetzten optischen Systems an seiner Gesammtleistung festzustellen. Das Fehlen eines sichern Leitfadens für eine derartige Feststellung, $d . h$. beim Mikroskop das Fehlen eines sachgemässen Begriffs von Objectiv- und Ocularwirkung, der unter Ausscheidung des UnwesentJichen und Zufälligen den eigentlichen Gegensatz in den Functionen beider Bestandtheile zutreftend zum Austruck brächte, ist die Ursache wesentlicher Mängel, welche der bisherigen Theorie des Mikroskops anhaften und auch die Veranlassung zu einigen Irrwegen, auf welche das Streben nach Vervollkommnung dieses Instruments gerathen ist.

Wenn man den Zweck des Objectivs in die Erzeugung eines reellen Bildes und den des Oculars in dessen weitere Vergrösserung setzt, so wird durch diese Erklärung - so richtig und nützlich sie ja ist - das wesentliche Princip des zusummengesetzten Mikroskops keineswegs getroffen. Es ist diess schon daran zu bemerken, dass dieser Angabe zufolge die Verbindung von Objectiv und Ocular: bloss der Vergrösserung zu dienen scheint, während doch die notorische Ueberlegenheit des zusammengesetzten Mikroskops gegenüber dem besten Simplex vielmehr in der Qualität der Leistung besteht und auch schon bei solchen Vergrösserungen zu Tage tritt, die sich ohne alle Schwierigkeit mit dem einfachen Mikroskop erreichen lassen. Worin dagegen die eigentliche Bedeutung des Princips der 
Zusammensetzung zu suchen sei, ergiebt der Hinweis darauf, dass beim Compositum in allen Fällen eine charakteristische Theilung der Arbeit in Bezug auf jene Functionen der Focalwirkung und der Flächenausbreitung vorliegt, in der Art, dass die specifischen Effecte der einen im Objectiv, der andern im Ocular ihren Sitz haben. Im Objectiv erfolgt die Flächenausbreitung des Bildes praktisch so gut wie rollkommen nach den Gesetzen für die Abbildung eines unendlich kleinen Flächenelements; im Ocular erfolgt die Focusverschiebung, d, h. die Divergenzänderung in den einzelnen Lichtbüscheln bis auf ummerkliche Abweichungen so wie an unendlich engen Strahlenbüscheln. Dagegen kommt dort das eigenthümliche Moment der Divergenzänderung von Strahlenkegeln grossen Oeffnungswinkels, hier das eigenthümliche Moment der Ausbreitung einer Bildfläche auf grossen Bildwinkel zur Geltung. Es lässt sich aber beweisen, dass die Erzeugung eines einigermassen vollkommenen Bildes unter den fraglichen Bedingungen über Oeffnungs- und Bildwinkel bei keinem optischen Apparat anders möglich ist als durch eine solche Vertheilung der specifischen Focalwirkung und der specifischen Vergrösserung auf besondere Bestandtheile des optischen Systems und dass mithin beim Mikroskop im Besondern die Höhe seiner Leistung wesentlich in dieser Seite des Zusammenwirkens von Objectiv und Ocular begründet ist. Dabei ergiebt sich indess, dass - wenigstens unter Voraussetzung des zur Zeit in Anwendung stehenden Constructionsprincips - die sachliche Grenzscheide zwiObjectiv- und Ocularfunction nicht da zu suchen ist, wo das reelle Bild des Objectivs dem Ocular zugefuhrt wird, sondern vielmehr da, wo im Objectiv die divergent eingetretenen Strahlenbüschel durch wiederholte Brechungen in parallelstrahlige Büschel umgewandelt sind - von wo aus sie durch eine weitere Brechung nach dem Ocular hin convergirend gemacht werden.

6. Die Consequenz dieser Resultate ist eine besondere Art schematischer Zerlegung des Mikroskops, welche an Stelle der jetzt ubblichen überall dann eintreten muss; wenn es darauf ankommt, die Qualität der mikroskopischen Bilder auf ihre maassgebenden Bedingungen zurückzuführen; welche aber auch als Grundlage für die Bestimmung der quantitativen Verhältnisse der Wirkung mit Vortheil gebraucht. werden kann. Ihr zufolge besteht der erste Act im Abbildungsvorgang nicht in der Erzeugung des umgekehrten reellen Objectivbildes vor oder in dem Ocular, sondern vielmehr in der 
Erzeugung eines, den parallelstrahligen Büscheln entsprechenden unendlich entfernten virtuellen Bildes; der zweite Act aber umfasst dessen fernere Abbildung unter dem Gesichtswinkel des Ocularfeldes und in der Weite des deutlichen Sehens and kommt durch eine letzte Brechung der Strahlen im Objectiv and durch die verschiedenen Brechungen im Ocular zu Stande. Den ersteren kann man die Lupenwirkung des Objectivs nemnen, weil dieser Theil der Leistung vollkommen identisch ist mit derjenigen einer gewöhnlichen Lupe für ein fernsichtiges Auge; der zweite Theil aber entspricht offenbar, alle einzelnen Veränderungen des Strahlengangs zusammengefasst, der Wirkung eines Fern ro hrs mit kleiner Objectivöffnung. welchem das vorher erwähnte unendlich entfernte virtuelle Bild als Object dient ${ }^{1}$ ). Diese Zerlegung der Gesammtwirkung des Mikroskops wird dadurch eine vollkommen bestimmte, dass der Sitz der letzten im Objectiv eintretenden Brechung, welche die parallelstrahligen Strahlenbüschel nach dem Ocular convergent macht, immer in die obere Focalebene des Objectivs verlegt werden kann. Eine an dieser Stelle aus letzterem ausgeschieden gedachte Linse von einer der Tubuslïnge entsprechenden Brennweite gibt alsdann das Objectiv des schematischen Fernrohrs, dessen wirksame A ngularvergrösserung nach bekannten Regeln durch Tubuslänge und Ocularstärke gegeben ist. Die Aequivalentbrennweite des die Lupenwirkung im Objectiv vermittelnden Systems bleibt dabei gleich derjenigen des Objectivs selbst und bestimmt in bekannter Weise den Gesichtswinkel, unter welchem das mikroskopische Object bei gegebenem linearen Durchmesser in dem unendlich entfernten Bilde erscheint.

Das hier dargelegte Ineinandergreifen von Objectiv- und Ocularfunction in Form von Lupenwirkung und Fernrohrwirkung muss als die allgemeingiltige Charaltteristik für das heute geltende Constructionsprincip des zusammengesetzten Mikroskops hingestellt werden. Wie das Folgende zeigen wird, beantworten sich auf Grund derselben zahlreiche für die Theorie des Mikroskops und für dessen rationelle Construction gleich wichtige Fragen - nach dem Sitz der verschiedenen Fehlerquellen, nach den Mitteln zu ihrer Beseitigung,

1) Zur Erlänterung soi darauf hingewiesen, dass in der That ein wirkliches Mikroskop entsteht, wenn eine correcte Lupe von beliebiger Brennweite centrirt vor dem Objectiv eines Fernrohrs angebracht wird. 
nach der Grenze der unter gegebenen Bedingungen möglichen Vollkommenheit, nach dem Einfluss, welchen Brennweite des Objectirs, Tubuslänge und Ocularstärke auf die Qualität des Gesammteffects üben, u. a. m.

\%. Im Vorstehenden sind die obersten Gesichtspunkte angezeigt, von welchen eine erschöpfende Theorie des Mikroskops, in ihrem rein dioptrischen Theil, ausgehen muss. Von ihnen aus ergibt sich eine Theorie der Abbildungsfehler oder Aberrationen, die den besondern Aufgaben gewachsen ist, wie die abnorme Grösse des Oeffnungswinkels beim Mikroskop sie mit sich bringt. Es zeigt sich, dass diese Abbildungsfehler in zwei selbständige Classen zerfallen; die eine umfasst die Fehler der Focalwirkung - Aberrationen im engern Sinne -, die andere wird gebildet durch Fehler der Flächenausbreitung (Vergrösserung), welche beim Mikroskop bisher ausser Acht geblieben sind. Zur ersten Art gehört die sphärische und chromatische Aberration, die man gewöhnlich betrachtet; die zweite begreift eine Reihe eigenthümlicher Abweichungen vom normalen Strablenlauf, welche sämmtlich daraus entspringen, dass die verschiedenen Theile eines die freie Oeffnung erfüllenden homofocalen Strahlenbüschels, nach Maassgabe der verschiedenen Neigung dieser Theile gegen die Axe und der ungleichen Brechbarkeit der einzelnen Earben, Bilder von ungleicher Vergrösserung liefern - ungleich, sowohl wenn die verschiedenen partiellen Bilder untereinander, wie auch wenn innerhalb je eines derselben verschiedene Richtungen im Sehfelde verglichen werden. Aus diesen Abweichungen, welche ich nicht Aberrationen, sondern A nomalien der Vergrósserung nenne, resultiren nicht nur die belzannten Unvollkommenheiten des mikroskopischen Bildes ausserhalb der Mitte des Sehfeldes, sondern auch eine besondere Art chromatischer Fehler, welche man, obwohl sie mit der eigentlichen Achromasie gar nichts zu thun haben, bisher immer als Merkmale chromatischer Focusdifferenzen gedeutet hat.

Die Abbildungsfehler der zweiten Classe bestimmen ausschliesslich die Beschaffenheit des Bildes ausserhalb der Axe; die Vollkommenheit der Strahlenvereinigung in der Mitte des Sehfeldes dagegen, und damit die maximale Leistungsfähigkeit des Mikroskops, ist allein von den wirklichen Aberrationen, der chromatischen und der sphärischen, abhängig. - Die genauere Analyse dieser ergiebt Folgendes:

Erstens. Die chromatische Aberration, wie sie bei grossem 
Oeffnungswinkel zur Geltung kommt, beruht nicht allein in denjengen Focusdifferenzen, welche - der Farbenabweichung selbst und ihrem ungleichförmigen Gang in Crown- and Flintglas entsprechend - die abbildenden Strahlenkegel im Ganzen treffen, sondern ebenso sehr in einer unvermeidlichen Ungleichheit der Farbenvereinigung für verschieden geneigte Strahlenbaischel innerhalb des Oeffnungswinkels, die sich darin äussert, dass ein für gerade Beleuchtung vollkommen achromatisches Objectiv für schief einfallendes Licht mehr oder minder ü ber corrigirt sein muss. Wührend die ersterwähnten gewöhnlichen (primären und secundären) Farbenabweichungen bei correcter Construction sich entweder ganz heben oder doch wenigstens fast unmerklich machen lassen, ist diese zweite Fehlerquelle mit den heute der Technik zu Gebote stehenden Materialien durch keine Kunst zu beseitigen. Ihr Einfluss aber ist gross genug, um wenigstens bei den mittleren und mässig starken Objectiven der erreichbairen Vollkommenheit schon da eine Schranke zu setzen, wo die andern unvermeidlichen Fehlerquellen solches noch nicht thun. Nach meinen Erfahrungen bleibt die thatsächliche Leistungsfähigkeit der Objective von $6-3 \mathrm{Mm}$. Brennweite allein in Folge dies es Umstandes merklich hinter derjenigen Höhe zurück, welche die mögliche Vollkommenheit im Punkte der sphärischen Aberration und die mögliche Vollendung in der technischen Ausführung andernfalls zulassen würden.

Zweitens. Die sphärische Aberration zerfällt bei einer strengeren Untersuchung ihrer Bedingungen in eine Reihe von selbstständigen Gliedern, die in ihrem Anwachsen mit der zunehmenden Neigung der Strahlen gegen die Axe einen sehr ungleichen Gang befolgen. Eine wirkliche Aufhebung ist nur für die beiden ersten Glieder theoretisch möglich. Sobald der Oeffnungswinkel über einen ganz geringen Betrag hinausgeht, kann die Ausgleichung der sphärischen Aberration nicht anders erfolgen als dadurch, dass die nicht aufhebbaren höheren Glieder durch absichtlich herbeigeführte Reste der niedern compensirt werden. Das Anwachsen des unvermeidlichen Deficits, das diese Compensation wegen des ungleĭchen Ganges der einzelnen Theile nothwendig übrig lässt, bestimmt die Grenze, welche dem Oeffnungswinkel gesetzt werden muss, wenn jenes Deficit im mikrospischen Bilde ohne schädliche Wirkung bleiben soll. - Für Oeffnungswinkel über $60^{\circ}$ hinaus, zumal aber für die grossen Beträge desselben bei den neueren starken Objectiven, 
ist die theoretisch unerlässliche Voraussetzung einer genügenden Compensation der bekannte Constructionstypus, nach welchem eine unachromatische, nahezu halbkugelige Frontlinse mit einer stark übercorrigirten Linsengruppe verbunden wird. Seine Erfindung (durch Amici?) muss als die eigentliche Grundlage aller neueren Fortschritte in der Vervollkommnung des Mikroskops angesehen werden.

Für das Trockensystem ergiebt sich eine ziemlich bestimmte Grösse der freien Oeffnung - 105 bis $110^{\circ}-$, über welche hinaus eine hinreichende Einschränkung der sphär. Aberration auf keine Weise mehr möglich ist, wenn nicht der freie Objectabstand des Mikroskops unter das äusserste fitr den Gebrauch unentbehrliche Mininum vermindert werden soll. - Die Anwendung der Immersion dagegen gewährt, bei richtiger Benutzung der hierdurch eingeführten neuen Verhältnisse, die Möglichleit, die Correction der sphär. Abweichung für sehr viel grössere Oeffnungswinkel genügend auszuführen, und zwar selbst für solche Beträge, welche das beim Trockensystem geometrisch mögliche Maximum $\left(180^{\circ}\right)$ noch etwas überschreiten. In dieser Möglichkeit sehr grosser Oeffnungswinkel, unbeschadet gleichmässiger Correction, und in der Verminderung der Lichtverluste, welche die Reflexion an der untersten Linsenfläche namentlich für die schief einfallenden Strahlen herbeiführt, liegen die einzigen wirklichen Vortheile der Immersionsmethode; Alles was man ausser diesen noch als Vorzüge derselben geltend gemacht lhat, beruht auf Missverständnissen. Das Folgende aber wird zeigen, dass diese beiden Unterschiede grade ausreichen, um die notorische Ueberlegenheit der Immersionssysteme vollkommen zu erklären.

Die mathematische Theorie liefert ferner einige praktisch wichtige Aufschlüsse über die Form, in welcher bei incorrecten Constructionen, welche ein merkliches Residuum sphärischer Aberration übrig lassen, dieses zur Erscheinung kommt. Wie verschieden auch im einzelnen Falle der thatsächliche Verlauf der Strahlen in der Nähe ihres schematischen Vereinigungspunktes sein mag, immer lässt sich derselbe durch blosse Veränderung einer Linsendistanz im System (wie z. B. die Deckglascorrection herbeiführt) so umgestalten, dass der centrale Theil und die äusserste Randzone des Objectivs richtig zusammenwirken, während die zwischenliegende mittlere Zone alsdann mehr oder minder übercorrigirt bleibt. Zugleich aber zeigt 
sich, dass kein äusseres Hilfsmittel eine solche typische Correctionsdifferenz, wo sie einmal vorliegt, beseitigen oder auch nur vermindern kann. Weil sie in den Krümmungs- und Brechungsverhältnissen der untersten Linsen des Objectivs wurzelt, leisten ihr gegenüber alle Vorrichtungen, durch welche man auf die Verbesserung der Aberrationen hat einwirken wollen - besondere Correctionsgläser oberhalb der Objective oder besondere Constructionen des Oculars - im günstigsten Falle nur dasselbe, was auch die Veränderung der Linsendistanzen durch eine Correctionsfassung möglich macht. Sie gestatten, den vorhandenen Aberratiousrest zwischen Mittel und Rand der freien Oeffnung gleichsam hin und her zu schieben und auf diese Weise irgend eine einzelne Zone des Objectivs auf Kosten der übrigen vorübergehend mehr oder minder aberrationsfrei zu erhalten. - Einrichtungen wie der von RoystonPig ot t ausgedachte maplanatic searcher " entspringen aus einer vollständigen Verkennung der wirklichen Sachlage. Sie stützen sich auf einen Begriff von sphärischer Aberration, der - weil er nur Spielraum lässt für die einfache Alternative: übercorrigirt oder untercorrigirt - mit sammt der ganzen darauf gebauten Theorie der aplanatischen Brenmpunkte gegeniiber den heutigen stärkeren Mikroskopen durchaus gegenstandlos ist. Durch solche Hilfsmittel wird die wirkliche Leistungsfähigkeit des Mikroskops niemals erhöht werden; denn Alles, was sie bewirken können, ist bei richtiger Construction auch schon im Objectiv selbst zu erreichen, und Alles, was hier nicht $\mathrm{zu}$ erreichen ist oder in einer verfehlten Construction nicht erreicht worden ist, kann auch durch sie nicht geleistet werden.

8. Ihren Abschluss findet die Analyse der Vollkommenheitsbedingungen in dem Nachweis des Einflusses, den die verschiedenen Bestandtheile des Milkroskops auf die Qualität des Gesammteffects ausüben. Hier ergiebt sich nun vor Allem, dass die für die Correctheit der Abbildung in der Mitte des Sehfeldes und damit für das mögliche Maximum der Leistung maassgebenden Factoren, nämlich die chromatische und die sphärische Aberration in dem oben bezeichneten bestimmteren Sinne, allein in der Construction der Objective wurzeln und dass die Einrichtung der Oculare anf jene überhaupt keinen irgend merklichen Einfluss gewinnen kann; dass aber ferner auch alle sonstigen Abbildungsfehler, zu welchen das Ocular theilweise mitwirkt, doch nur insoweit die erreichbare Vollkommenheit der Gesammtleistung begrenzen, als unvermeidliche 
Reste jener in der Wirkung des Objectivs, und zwar in demjenigen Theile derselben, der oben als specifische Focalwirkung bezeichnet wurde, übrig bleiben. Von groben Verstössen in der Construction natürlich abgesehen, kann der ganze Ocularapparat des Mikroskops, g'egenüber den in der Focalwirkung des Objectivs begründeten Aberrationen und Vergrösserungsanomalien praklisch als vollkommen fehlerfrei angesehen werden, und zwar in allen wesentlichen Punkten auch dann, wenn nur die einfachsten unter den bekannten Oculareinrichtungen in Anwendung lommen. Hieraus folgt, das die mögliche Höhe der Leistung beim Mikroskop allein in der Construction der Objective wurzelt und dass keine denkbare Vervollkommnug der Oculare sie im Geringsten beeinflussen kann; ferner aber, dass auch die besondern Umstände, unter welchen der Ocularapparat fungiren mag, namentlich die Art, wie die Vergrösserung durch die Länge des Tubus und die Stärlse des Oculars bewirkt wird, innerhalb des praktisch in Betracht kommenden Spielraums vollkommen gleichgültig bleiben, und - richtige Anpassung der Objective an die einmal angenommenen Verhältnisse vorausgesetzt - die erreichbare Höhe der Leistung durchaus nicht berühren. Was in England zu Gunsten des langen Tubus vorgebracht wird, ist theoretisch ebenso unhaltbar, wie Dasjenige, was neuerdings — von Praszmows ki als Vortheil des kurzen Tubus hingestellt worden ist; wie sich denn diese vermeintlichen Unterschiede auch thatsächlich als nicht vorhanden ergeben, sobald man unter wirklich vergleichbaren Bedingungen sie beobachten will. Desgleichen lassen Theorie und accurate Experimente übereinstimmend Alles als reine Einbildung erkennen, was über die ausserordentlichen Leistungen dieser oder jener besondern Ocularconstruction berichtet wird - so weit es sich dabei um wirkliche Steigerung des optischen Vermögens, nicht etwa bloss um nebensächliche Vortheile (Grösse des Sehfeldes u. dergl.) handeln soll.

Im Hinblick auf diese Resultate gewinnt die oben erwähnte Grenzbestimmung in Bezug auf Objectiv- und Ocularfunction beim Mikroskop und das auf sie gegründete Zerlegungschema eine besondere Tragweite. Alle Abbildungsfehler, die überhaupt die Wirkung beeinflussen, finden ihren vollständigen Ausdruck schon in der Beschaffemheit des unendlich entfernten virtuellen Bildes, welches das Objectiv, ì la Lupenwirkung, vom Object erzeugt; diesem 
gegenüber spielt der ganze Ocularapparat, wie er sich aus Tubus und Ocularlinsen zusammensetzt, die Rolle eines indifferenten Vergrösserungs - Mechanismus, der - à la Fernrohr wirkend - nur dazu dient, jenes Objectivbild dem beobachtenden Auge auf den erforderlichen Sehwinkel auszubreiten, ohne dabei seinem Inhalte irgend etwas hinzuzufügen oder irgend etwas von ihm hinwegzu* nehmen. Dieser Inhalt selbst aber ist, seinem mög li ch en Detail nach, bestimmt durch die angulare Grösse der Zerstreuungskreise, welche die in der Construction des Objectivs begründeten Abbildungsfehler an Stelle scharfer Bildpunkte in das Lupenbild einführen. Indem man deren Eingreifen in den schliesslichen Effect in Betracht zieht, ergiebt sich für jedes concrete Objectiv eine durch Tubuslänge und Ocularstärke beliebig zusammensetzbare Angularvergrösserung, welche für ein Auge von angenommener normaler Sehkraft grade a usreichen muss, das im Objectivbild möglicher Weise abbildbare Detail vollkommen zu erkennen. Diese - welche man die förderliche Angularvergrösserung nennen kann - muss als das Maass der relativen Vollkommenheit des Objectivs angesehen werden; aus ihr bestimmt sich in leicht zu erkennender Weise mit Hilfe seiner Brennweite die förderliche Limearvergrösserung, d. h. diejenige Vergrösserungsziffer, mit welcher die Leistung des betreffenden Objectivs erschöpft ist; es ist die kleinste Vergrösserung, bei der man alles Detail sieht, welches mit ihm, seiner dioptrischen Vollkommenheit nach, überhaupt abgebildet werden krann. Eine stärkere Vergrösserung kann zwar noch brauchbar sein, indem sie solches Detail deutlicher und bequemer zur Wahrnehmung bringt; sie vermag aber niemals das optische Vermögen eines gegebenen Objectivs zu erhöhen. - Unter Voraussetzung gleicher relativer Vollkommenheit der Construction muss für Objective der verschiedensten Brennweiten die angulare Grösse der Zerstreuungskreise in ihren Lupenbildern ein und dieselbe sein; es muss also die absolute Grösse der kleinsten Theile, die noch getrennt abgebildet werden, ein und denselben Bruchtheil der Brennweite ausmachen. Daraus folgt einerseits, dass die förderliche Angularvergrösserung für solche objective gleich ist und inre Hohe demnach das Maass für die relative Vollkommenheit darstellt; andererseits, dass die förderliche Linearvergrösserung, mithin das absolute optische Vermögen, bei gleicher relativer Gute der Construction in demselben Verhältniss zunehmen muss, in welchem die Brennweits abninmt. 
D. Was die praktische Anwendung der hier aufgestellten Definitionen anlangt, so darf nicht übersehen werden, dass die aus den Aberuationsresten und den Mängeln der technischen Ausführung herlührenden Zerstreuungskreise im Lupenbild der Objective beim wirklichen Gebrauch stärkerer Objective niemals mit demjenigen Betrage zur Geltung kommen, der einem die ganze freie Oeffnung erfüllenden Strahlenkegel entsprechen würde. Thatsächlich wird, sobald der Oeffnungswinkel eine beträchtliche Grösse besitzt, immer nur ein Theil desselben von den abbildenden Strahlenbüscheln gleichzeitig in Anspruch genommen, daher denn auch nur dieser Theil die effectiv werdenden Aberrationen veranlasst; and da nun - wie die Beobachtung der Oeffnungsbilder lehren kann - der für die Abbildung thätige Theil der freien Oeffnung nach Grösse und Lage fortwährend wechselt, je nach der Art der Beleuchtung und der Structur der Präparate, so folgt, dass von einer allgemeingiltigen Bestimmung der förderlichen Vergrösserung oder des optischen Vermögens nicht die Rede sein kann ${ }^{1}$ ). Nichtsdestoweniger sind die hier geltend gemachten theoretischen Gesichtspunkte zu einer beiläufigen Abschätzung der Leistungen, welche vom Mikroskop heut zu Tage erwartet werden dürfen, vollkommen brauchbar und ihr Hervorkehren ist sehr geeignet, grosse Illusionen zu beseitigen, denen sich manche Mikrographen in Bezug auf diese Leistungen augenscheinlich hingeben. Die theoretische Discussion der Abbildungsfehler, ihr allseitiges praktisches Studium mittels der im

1) Auch die von Harting zuerst empfohlene Methode, die Unterscheidungsgrenze der Mikroskope an den kleinen optischen Bildchen, wie man solehe durch Luftblasen u. dergl. erhalten kann, zu bestimmen, gewährt eine allgemeingiltige und praktisch verwerthbare Feststellung des optischen Vermögens nicht. Denn abgesehen davon, dass bei ihrer Anwendung der wirk. sam werdende Strahlengang weit abliegt von allen denjenigen Formen, dio beim normalen Gebrauch, wenigstens der stärkeren Objective, thatsächlich vorkommen, lässt sich auch auf die im folgenden Abschnitt zu gebenden Nachweise hin darthun, dass die Unterscheidung des Details in solchen Luftblasenbildchen keineswegs von der dioptrischen Wirkung der Objective allein, sondern ebensosehr von besondern, ganz selbständigen Einflüssen ausserhalb des Mikroskops abhängt. Die Resultate jener Methode geben thatsächlich nur die Grenzen eines der dioptrischen Vollkommenheit fremden Auflösungsvermögens, ganz so wie die Beobachtungen an der Norbert'schen Probeplatte und an den Diatomeen, nur unter etwas veränderten Beleuchtungsver. hältnissen. 
Folgenden erwähnten Methode und die sorgfältige Prüfung einer ziemlichen Anzahl von Objectiven neueren Datums aus den berühmtesten Werkstätten diesseits und jenseits des Canals haben mich übereinstimmend zu dem Schlusse geführt, dass die heute erreichten und erreichbaren Ziffern förderlicher Vergrösserung durchweg sehr viel geringer sind, als man nach der Freigebigkeit mancher Mikroskopiker mit Tausenden und Zehntausenden denken sollte. Nach meinen Erfahrungen wird unter Voraussetzung der gewöhnlichen Beleuchtungsformen - abgesehen also von ganz abnormen Verhältnissen des Strahlenganges, die sich etwa mit directem Sonnenlicht herstellen lassen, praktisch aber so gut wie werthlos sind - auch bei den allervollkommensten Systemen die optische Capacität schon bei höchstens 8facher Angularvergrösserung erschöpft, so dass Alles im Lupenbild überhaupt abbildbare Detail einem normalen Auge auf jeden Fall zugänglich ist, wenn Tubus und Ocular zusammen ein 8fach vergrösserndes Fernrohr darstellen. Aber selbst diese Leistung wird nur bei den schwächeren und mittleren Objectiven erreicht. Wenn die Brennweite unter $3 \mathrm{Mm}$. herabgeht, wird die relative Vollkommenheit der Constructionen wegen der rasch wachsenden technischen Schwierigkeiten merklich geringer; und ich bin sicher, dass es kein Objectiv unter $1 \mathrm{Mm}$. Brennweite giebt, dessen optisches Vermögen über eine 5fache Angularvergrösserung hinausreicht. Man kann leicht ausrechnen, welche Ziffern sich hiernach für die förderliche Linearvergrösserung bei den verschiedenen Brenmweiten ergeben (ca. 500 bei $4 \mathrm{Mm}$., ca. 1200 bei $1 \mathrm{Mm}$.) und dann weiter, welche Vergrösserungen man äussersten Falls noch als nützlich und brauchbar wird anerkennen dürfen, wenn man beachtet, dass die, blosse Ausdehnung des Bildes auf grösseren Sehwinkel ohne entsprechende Steigerung des optischen Vermögens, namentlich bei an sich schon hohen und lichtschwachen Vergrösserungen, der Deutlichkeit der Wahrnehmung bald viel mehr schaden als nützen muss.

Is ist hieraus zu entnehmen, wie gegenstandslos alle Bemühungen sind, welche darauf ausgehen, die Vergrösserung des Mikroskops durch besondere Construction des Ocularapparats ins Ungemessene zu steigern. Was aber die Hoffnung anlangt, durch Verkürzung der Brennweiten der Objective die Leistungsfähigkeit des Instruments mit der Zeit immer weiter erhöht $\mathrm{zu}$ sehen, so tritt dieser - von mehreren sonstigen Bedenken abgesehen - ein Hinderniss entgegen, welches nach dem dermaligen Stand unseres 
Wissens als ein absolutes hingestellt werden muss. $\mathrm{Zu}$ je hoheren Vergrösserungen man nämlich fortgeht, desto mehr wird die aus den. Aberrationsresten im Objectiv und aus seinen technischen Mängeln entspringende Unvollkommenheit des Bildes vergrössert durch den Effect der Diffraction, welche die Kleinheit der freien Oeffnung bei den starken Objectiven einführt. Diese Diffractionswirkang der Linsenöflnung ${ }^{1}$ ) verwandelt gleichfalls das Bild jedes Objectpunktes in einen Zerstreuungskreis von grösserem oder geringerem Durchmesser; während aber die hieraus lesultirende Verminderung des optischen Vermögens bei nöässigen Vergrösserungen unmerklich bleibt gegenüber dem Effect der Aberrationsreste, fä,llt sie bei hohel Ziffern ausserordentlich in's Gewicht. Es lässt sich allgemein beweisen, dass der Eintluss dieser von der optischen Vollkommenheit ganz unabhängigen Fehlerquelle beim Mikroskop (wie auch beim Fernrohr) allein durch die Grösse des letzten Oeffnungsbildes im Augenpunkt des Oculars bedingt und zwar dieser Grösse umgekehrt proportional ist; er gestaltet sich in allen Stücken genau so, wie. wenn das von ihm noch frei gedachte mikroskopische Bild dureh ein körperliches Diaphragma, dessen lichter Durchmesser dem jenes Oeffnungsildes gleich ist, betrachtet, würde. Dieser Durchmesser aber hängt allein vom Oeffnungswinkel des Mikroskops und seiner Gesammtbrennweite, also seiner Gesammtvergrösserung, ab, und kann nach der unter (4) gegebenen Norm für jeden Fall berechnet werden. Diejenige Grösse des Oeffnungswinkels vorausgesetzt, die auch beim Immersionssystem nicht wesentlich iiberschritten werden kaun, - $180^{\circ}$ in Luft - findet man z. B. für 1000 fache Vergrösserung $0,5 \mathrm{Mm}$., für 5000 fache $0,1 \mathrm{Mm}$. u. s. f., unabhängig davon, wie diese Vergrösserungen durch Objectiv und Ocular hervorgebracht sein mögen. Und wemn man nun wissen will, welche Bedingungen von dieser Seite her z. B. einer 5000fachen Vergrösserung gestellt sind, so braucht man nur in ein Staniolblättchen einen Nadelstich von $0,1 \mathrm{Mm}$. Durchmesser za machen und durch das so erhaltene Diaphragma hindurch einen gut begrenzten hellen Gegenstand, etwa eine Lichtflamme, anzusehen; man hat dabei unmittelbar vor Augen,

1) Sie ist wohl zu unterscheiden von den im folgenden Abschnitt zu besprechenden Diffractionserscheinungen, welche die Structur der Objecte hervorruft. 
wie die Contouren eines mikroskopischen Objects unter jener Vergrösserung sich ausnehmen müssen, auch wenn das Mikroskop an sich, d. h. vom Beugungseffect abgesehen, a b s o lut vollkommen wäre.

Die Erwägung dieser Umstände muss za dem Eirgebniss führen, dáss eine erhebliche Steigerung des absoluten optischen Vermögens der Mikroskope über die heute mit Brennweiten von ca. $1 \mathrm{Mm}$. erreichbare Höhe hinaus auch in Zukunft nicht $\mathrm{zu}$ erwarten steht, weder von einer Verkïrzung der Brennweiten noch von einer weitern Vervollkommnung der Constructionen. Wie es heute kein Mikroskop giebt, dessen nutzbare Vergrösserung - wenn man es mit diesem Attribut ernsthaft nimmt - auch nur 4000 erreichte, so wird es auch in Zukunft keines geben. Die Darlegungen in den letzten beiden Abschnitten aber werden zeigen, dass selbst Vergrösserungen weit unter der Hälfte dieser Ziffer, die sich mit Objectiven von $1 \mathrm{Mm}$. Brennweite als nutzbare recht gut erhalten lassen, thatsächlich schon nicht mehr verwerthbar sind, weil anderweitige, vou der dioptrischen Vollkommenheit der Abbildung unabhängige Bedingungen für eine solche Verwerthung nicht mehr erfüllt werden können. Alles diess zusammengenommen begründet meinen Schluss : dass heut zu Tage die Vervollkommnung des Nikroskops überhaupt nicht mehr die Erlangung höherer förderlicher und höherer nutzbarer Vergrösserungen, also uberhaupt nicht mehr die Steigerung des absoluten optischen Vermögens zum Ziele hat; dass vielmehr dieses ausschliesslich in die Erhöhung der Leistungsfähigkeit der mittleren and mässig starken Objective zu setzen sei." - Es wird einen wirklichen, dem wissenschaftlichen Gebrauch des Mikroskops in hohem Grade nützlichen Fortschritt der optischen Kunst bezeichnen, wenn es gelingt, mit Objectiven von $3-4$ Mm. Brennweite Dasjenige zu leisten, was gegenwärtig nur mit sehr viel stärkeren Linsen erreicht werden kann. Das liegt im Bereiche des Möglichen; andere Ziele gehen auf Luftschlösser aus.

10. In der angefiihrten ausfïhrlicheren Darstellung meiner Untersuchungen werde ich neue und sehr exacte Methoden mittheilen, nach welchen die sämmtlichen Bestimmungsstiucke eines Mikroskops ".... die Brennweiten der einzelnen Bestandtheile, der Oeffnungswinkel, die Maasse für Objectiv- und Ocularwirkung etc. - empirisch ormittelt werden können; daneben aber werde ich auch ein Verfahren beschreiben, welches erlaubt, sämmtliche durch die 
Theorie nachweisbare Abbildungsfehler mit sehr einfachen Hilfsmitteln am fertigen Instrument $z u$ beobachten und daraufhin den Grad seiner Vollkommenheit zu bestimmen. - Die gewöhnlich empfohlenen Methoden zur Prüfung der sphärischen und der chromatischen Correction der Objective sind den thatsächlichen Verhältnissen durchaus nicht gewachsen und weit davon entfernt, zu einer auch nur einigermaassen vollständigen Kenntniss jener Fehlerquellen an fertigen Instrumenten zu führen. Denn die durch jene Methoden sichtbar zu machenden Effecte der Abbildungsfehler sind nicht elementare Wirkungen, sondern Gesammteffecte aus vielen verschiedenartigen Ursachen. Bei dem sehr ungleichen Gewicht, welches den einzelnen unter ihnen, nach ihrem Einfluss beim wirklichen Gebrauch des Mikroskops, zukommt, muss das Urtheil nach dem Totaleffect unter Umständen völlig fehlgreifen. Die zutreffende Würdigung eines Objectivs in Bezug auf seine Vollkommenheit und Leistungsfähigkeit kann vielmehr - wenn sie überhaupt auf den Befund über die Abbildungsfehler gegründet werden soll - nur durch die Zerlegung der sichtbaren Abweichungen in ihre Bestandtheile und den Nachweis der sämmtlichen wirksamen Fehlerquellen im Einzelnen gewonnen werden.

Da die Beschreibung der Prüfungsmethode, welche dieses leistet, nicht vollständig gegeben werden kann ohne eine specielle Discussion der sämmtlichen Abbildungsfehler und genaue Angaben über ihre Erscheinungsformen, so sei hier nur das Princip des Verfahrens im Allgemeinen bezeichnet. - Als Object dient dabei ein Präparat, welches nichts anderes als scharfe Grenzen zwischen vollkommen durchsichtigen und ganz oder fast ganz undurchsichtigen Theilen in nerhalb einer einzigen Ebene darbietet und keinerlei merkliche Ablenkungen an den hindurchtretenden Strahlen hervorbringt. Man erhält ein solches in einer für alle Zwecke ausreichenden Vollkommenheit, indem man (mittelst der Theilmaschine) gröbere und feinere Liniengruppen in eine Silber- oder Goldschicht einritzt, wie man solche in einer Dicke, die nur einen kleinen Bruchtheil des MikroMm. ausmacht, nach bekannten Methoden auf Glas niederschlagen kann; Deckgläser von verschiedener, genau gemessener Stärke, auf ihren untern Flächen mit solchen Theilungen $(10-50$ Linien pro MIm.) versehen, werden nebeneinander mit Balsam auf einen Objectträger gekittet; Ein Präparat dieser Art dient für die stärksten wie für die schwächsten Objective. $\mathrm{Zu}$ seinem Gebrauch gehört der Beleuchtungsapparat, welcher in dem folgenden Aufsatze dieses 
Heftes beschrieben ist, oder irgend eine Vorrichtung, die gestattet, das Präparat von mehreren lichtstrahlenden Flächen aus g lei chzeitig zu beleuchten und den Verlauf der Strahlenbüschel, welche cliese liefern, innerhalb des Oeffinungswinkels eines zu prüfenden Objectivs beliebig zu reguliren. Mit dem erwähnten Beleuchtungsapparat wird dieser Zweck auf das einfachste erreicht, indem man Diaphragmen mit mehreren getrennten Oeffnungen - aus Cartonpapier zugeschnitten - neben den gewöhnlichen Blendungen verwendet; unter Controle des Oeffnungsbildes in der oberen Focalebene des Objectivs kann man durch Blendungen mit passenden Ausschnitten bei Benutzung der Vorrichtungen zur Veränderung des Lichteinfalls ganz beliebige Theile des Oeffnungswinkels gleichzeitig in Thätigkeit setzen.

Das Verfahren der Prüfung zielt darauf $a b$, das Zusammenwirken der sämmtlichen Zonen der Objectivöffnung in der. Mitte und am Rand des Schfeldes zur Anschauung zu bringen und dabei dennoch die Bilder, welche sie einzeln gewähren, deutlich unterscheidbar zu erhalten. Zu dem Zweck wird die Beleuchtung so regulirt, dass in den Spuren der eintretenden Strahlenkegel in der obern Focalebene alle Zonen der freien Oeffnung, jede aber nur durch einen schmalen Streifen, repräsentirt sind, während diese Spuren selbst möglichst weit von einander abstehen. Je nach der Grösse des Oeffnungswinkels werden zwei oder drei isolirte Strahlenbüschel verwandt. Sie werden so angeordnet, dass, wenn z. B. die OeffnungsHäche des Objectivs $6 \mathrm{Mm}$. linearen Durchmesser besitzt, im ersteren Falle die -... nahezu kreisförmige - Spur des einen von der Mitte bis auf ca. 1,5 Mm. Abstand, die des andern a u f der entgegengesetzten Seite von der. Axe von 1,5 bis auf $3 \mathrm{Mm}$., d. h. bis zum Rand, sich erstreckt; im zweiten Falle aber der erste die Zone von der Axe aus bis zu $1 \mathrm{Mm}$. Abstand, der zweite die Zone zwischen $1 \mathrm{Mm}$. und $2 \mathrm{Mm}$. auf cler entgegengesetzten Seite und der dritte die Randzone zwischen 2 und $3 \mathrm{Mm}$., wieder auf der Seite des ersten, in Thätigkeit setzt. Diese Anordnung giebt den e mpfindlichen Strahlengang, bei welchem alle Correctionsmängel am stärksten zur Geltung kommen, weil die verschiedenen Strahlenkegel im Bilde unter möglichst grossen. Winkeln zusammentreffen. - So viel isolirte Theile der Oeffnungsfläche wirksam werden, so viel unterscheidbare Bilder erhält man von einer das Gesichtsfeld ausfüllenden Liniengruppe des Präparats. Bei einem in allen Stäcken 
absolut vollkommenen Objectiv müssten alle diese bei einer bestimmten Einstellung in ein einziges scharfes und farbenfreies Bild zusammenfliessen. Die Abbildungsfehler, soweit sie von der Art der sphärischen Aberration sind, haben aber zur Folge, dass eine delartige vollständige Verschmelzung der zwei oder drei partiellen Bilder wenigstens nicht durch das ganze Sehfeld zu Stande kommt und, soweit sie von der Farbenzerstreuung herrühren, dass die hellen Linien auf dunklem Grunde, sowohl die getrennten Bilder je einer wie auch die correspondirenden Bilder von mehreren an andern Stellen des Gesichtsfeldes die verschiedensten Farbensäume erhalten. Ein derartiges Probebild legt den ganzen Correctionszustand eines Mikroskops in allen Einzelheiten gleichzeitig vor Augen. Mittelst der Anleitung, welche die Theorie zur Diagnose der verschiedenen. Abbildungsfehler giebt, genügt die Vergleichung der Farbensäume der einzelnen partiellen Bilder, die Beobachtung ihres lateralen Auseinandertretens und inrer Niveaudifferenzen, in der Mitte des Sehfeldes und in den vier Quadranten der Randzone, alle Correctionsmängel in ihren letzten Bestandtheilen nach Art und Grösse genau zu definiren. Dabei erhält man namentlich Dasjenige, was von den eigentlichen Aberrationen - den Fehlern cler Focalwirkung - herrührt, deutlich getrenut von solchen Unvollkommenheiten; welche aus blossen Differenzen der Vergrösserung zwischen ungleich geneigten and ungleich brechbaren Strahlen - den Anomalien - entspringen und kann ausserdem durch eine einfache Manipulation den Einfluss des Oculars auf die Beschaffenheit des Bildes ausserhalb der Axe vollständig eliminiren ${ }^{1}$ ).

Die erforderliche theoretische Orientirung und praktische Erfahrung vorausgesetzt, un den Befund bei einer derartigen Prüfung in allen Punkten richtig würdigen zu können, gewährt das angedeutete Verfahren ein so erschöpfendes Urtheil über die Beschaffenheit eines Objectivs, dass darauf hin, wenn Brennweite und Oeffnungswinkel nebenbei ermittelt werden, seine Leistungsfähigkeit nach

1) Beispielsweise sei erwähnt, dass diese Methode, in Uebereinstimmung mit der Theorie, in der Farbenabweichung, welche bei grossem Oeffnungswinkel an jeder Stelle des Sehfeldes ausserhalb der Axe wirksam wird, nicht weniger als fü $\mathrm{n}$ f verschiedenartige Bestandtheile erkennen lässt, die wegen ihrer sehr ungleichen praktischen Bedeutung bei der Beurtheilung eines Mikroskops genau unterschieden werden müssen. 
allen Richtungen hin voraus zu bestimmen ist; wie ich mich durch zahlreiche Proben überzeugt habe. Für die gewöhnlichen Bedürfnisse der Mikroskopiker wird zwar die directere Prüfung mittelst natürlicher Probeobjecte stets den Vorzug behalten; immerhin dürfte die gelegentliche Anwendung dieser andern Methode nützliche Anhaltspunkte liefern, um die Ansprüche, welche man zur Zeit an die Beschaffenheit der Mikroskope stellen und nicht stellen darf, richtig bemessen zu lernen. Wer eimmal gute und im praktischen Gebrauch bewährte Objective auf diese Weise untersucht hat, wird ebensosehr gegen all zu naive Vorstellungen über ihre Vollkommenheit wie auch dagegen gesichert sein, Ansprüche zu erheben, welchen bis jetzt noch Niemand genügt hat:

11. Schliesslich soll noch in der Kürze derjenigen Sätze gedacht werden, welche die Wirkungsart der Beleuchtungsapparate und die fiur die Lichtstärke des Mikroskops maassgebenden Verhältnisse feststellen. In Bezug auf das Erstere trifft meine Betrachtungsweise nach ihren Ausgangspunkten und in ihren wesentlichen Resultaten mit denjenigen Lehren zusammen, durch welche $\mathrm{Nägeli}$ und Schwendener ${ }^{1}$ ) die Theorie der Beleuchtungsapparate, die seit Brewster and Wollast on die partie honteuse der mikrographischen Doctrin gewesen ist, zuerst auf sichere und deutliche Begriffe gebracht haben. Die Hauptsache besteht in Folgendem.

Die Beleuchtung der milkroskopischen Objecte bei der Beobachtung in durchfallendem Lichte exfolgt, was auch der Beleuchtungsapparat sein mag - Planspiegel, Hohlspiegel, Sammellinse, Condensor - - stets von einer begrenzten Fläche aus, welche, indem ihre Theile Strahlen einer primären Lichtquelle durch Spiegelung oder Brechung auf das Präparat leiten, diesem gegenüber wie selbstleuchtend sich verhält, und zwar - abgesehen von den Lichtverlusten durch Spiegelung und Brechung - selbstleuchtend mit der wirklichen Leuchtkraft der primären Lichtquelle. Die Grösse und die Lage dieser wir k sa men Lichtfläche (der Fläche des Hohlspiegels z. B.) bestimmt für jeden Punkt des Präparats einen kegelförmigen Raum, dessen Fortsetzung nach dem Mikroskop hin den Strahlenkegel ergibt, der von jeder durchsichtigen Stelle des Objects aus divergirend in das Objectiv eintritt, soweit derselbe innerhalb des Wiukelraums der freien Oeffnung fällt und soweit er

1) Das Mikroskop, Loipzig 1867. pag. $85 \mathrm{ff}$. 
nicht durch irgend welche Ablenkungen im Präparat ganz oder theilweise in andere Richtungen gelenkt wird, Die Spur dieses Strahlenkegels und der etwa aus ihm abgelenkten Strahlen erscheint als Bild der lichtgebenden Fläche (z. B. des Spiegels) in der obern Focalebene des Objectivs wie auch im Augenpunkt des Oculars, und kanu daselbst direct beobachtet werden; wobei u. A. der illusorische Charakter der angenommenen Untersehiede von convergirender, divergirender und parallelstrahliger Beleuchtung sinnenfällig hervortritt. - Kein noch so künstlicher Beleuchtungsapparat (Condensor) giebt jemals eine intensivere Beleuchtung als die primäre Lichtquelle unmittelbar geben könnte, wenn man -sie dem Objecte hinreichend nahe bringen würde. Kein solcher Apparat kann also einen andern Zweck wirklich erreichen, als den, mit Hilfe einer Lichtquelle von gegebener Lage und gegebener Ausdehnung eine beliebig gelegene und beliebig begrenzte mittelbar leuchtende Fläche von einer - bis auf die Lichtverluste - gleichen Leuchtlrraft unter dem mikroskopischen Präparat herzustellen.

Als praktische Anwendung dieser Sätze ergiebt sich eine Bèlenchtungsvorrichtung, welche sich von anderweit bekannten Apparaten dieser Art - sogen. Condensoren - dadurch unterscheidet, dass sie nicht den Anspruch macht, Wirkungen erzielen zu wollen, die theoretisch unmöglich, desshalb sicher auch nur in der Einbildung vorhanden sind, und welche dafür diejenigen Vortheile, die ein complicirterer Apparat gegenüber dern gewöhnlichen Beleuchtungsspiegel überhaupt darbieten kann, mit einfachen Mitteln wirklich in Geltung bringt. (S. den folgenden Aufsatz in diesem Heft).

12. Die auf die Lichtstärke des Mikroskops bezitglichen Fragen endlich finden ihre erschöpfende Antwort durch das folgende allgemein erweisbare Theorem:

Wenn von den Lichtverlusten durch Reflexion an den Linsen des Mikroskops abgesehen wird, is t die Helligkeit der vollkommen oder partiell durchsichtigen Theile eines Präparats im mikroskopischen Bilde genau diejenige, mit welcher sie beim directen Seben erscheinen wïrden, wenn vor die Pupille des Auges ein Diaphragma gestellt wäre, dessen freie Oeffnung congruent ist dem Bilde der lichtgebenden Fläche (des Spiegels z. B.), so wie es im letzten Oeffnu gisbild über dem Ocular des Mikroskops entworfen wird, - dabei das Präparat, in seiner 
Flächenausdehnung beliebig vergrössert, gegen die primäre Lich tquelle als Hintergrund gesehen vorausgesetzt.

Als unmittelbare Folgerungen hieraus ergeben sich folgende Sätze :

Die Helligkeit des mikroskopischen Bildes kann unter keinen Umständen diejenige übersteigen, mit welcher das Object dem freien Auge erscheinen würde. Für jeden bestimmten Winkeldurchmesser des abbildenden Strahlenkegels, sei nun dieser durch die freie Oeffnung des Milroskrops oder durch den Umfang der lichtgebenden Fläche hegrenzt, giebt es aber eine bestimmte Vergrösserungsziffer, unterhalb welcher die Helligkeit des Bildes der des direct gesehenen Objects stets gleich ist. Es ist diejenige Vergrösserung, bei welcher der Durchmesser des Bildes im Augenpunkt des Mikroskops dem der Pupille grade gleich wird.

So lange der Oeffnungswinkel des Objectivs kleiner ist als derjenige des zur Beleuchtung dienenden Strahlenkegels, wächst die Lichtstärke mit dem Oeffnungswinkel des ersteren und ist unabhängig von dem des letzteren; sobald aber - wie bei allen stärkeren Systemen der Fall - das Mikroskop einen grösseren Oeffnungswinkel hat als der vom Beleuchtungsapparat ausgehende Lichtkegel, vertauschen sich die Rollen beider.

Unter sonst gleichen Umständen hängt die Helligkeit der Bilder allein von der Vergrösserungsziffer $a b$, wofern das erwähnte Bild der lichtgebenden Fläche im Augenpunkt des Oculars kleiner als die Pupillenöffinung des Auges geworden ist; jene ist alsdann dem Quadrate der linearen Vergrösserung umgekehrt proportional. Im Besondern bleibt die Lichtstärke bei einer bestimmten Vergrösserung unabhängig davon, $o b$ diese durch ein starkes Objectiv und ein schwaches Ocular, oder umgekehrt, erzielt ist - wie sich durch ein einfaches ganz stringentes Experiment auch empirisch darthun lässt. Ebenso sind alle Objective, deren Oeffnung den grössten möglicher Weise zur Verwendung kommenden Divergenzwinkel der einfallenden Strahlen (40-50 Grad) uiberschreitet, wie verschieden auch ihre sonstige Vollkommenheit sein mag, in Hinsicht auf die Lichtstärke (bis auf praktisch unmerkliche Differenzen in den Lichtverlusten) einander sämmtlich glei ch, sobald sie unter gleicher Gesammtvergrösserung benutzt werden. - Die vermeintlichen Thatsachen, welche diesen letzteren Folgerungen entgegenzustehen scheinen, erklären sich daraus, dass alle Unterschiede in 
der Schärfe und Dentlichkeit der Bilder unwillkürlich und unbewusst als Unterschiede der Helligkeit gedeutet werden.

\section{Die physikalischen Bedingungen für die Abhildung feiner Structuren.}

13. Dass die Leistungen des Mikroskops nicht in allen Fällen von der geometrischen Vollkommenheit der Bilder allein abhängen, sondern, gewissen Classen von Objecten gegenüber, ausser durch jene noch durch die Grösse des Oeffnungswinkels bedingt seien, ist eine seit lange anerkannte Thatsache, deren Gewicht auch die Construction der Mikroskope in neuerer Zeit stark beeinflusst hat. Was aber die eigentliche Bedeutung dieser Thatsache sei; ist nichtsdestoweniger ebenso problematisch geblieben, wie das Wesen der besondern Capacität (Auflösungs- oder Unterscheidungsvermögen), welche man darauf hin dem Mikroskop hat beilegen müssen. Namentlich aber blieb fraglich, wie hoch der Nutzen dieses an den Oeffnungswinkel geknüpften Vermögens für den allgemeinen wissenschaftlichen Gebrauch des Instruments anzuschlagen sei und ob seine Tragweite überhaupt hinausreiche über gewisse specielle Vorkommnisse, bei welchen Schatteneffecte durch schiefe Beleuchtung vermuthet wurden. - Bei meinem Versuch, die Construction des Mikroskops auf die Theorie zu gründen, musste ich mir ganz besonders angelegen sein lassen, die Ansprüche festzusetzen, welehe im Interesse der allgemeinen, normalen Leistungen an den Oeffnungswinkel der Milkroskope zu stellen sind, wenn ich nicht Gefahr laufen wollte, einer blossen Tradition folgend, meine Arbeit vielleicht auf Ziele von sehr problematischem Werth zu richten. Wie ich glaube, werden denn auch die einschlagenden Fragen durch die nachfolgenden Ergebnisse insoweit definitiv erledigt, als nicht durch diese Ergebnisse selbst neue Thatsachen in den Gesiehtskreis treten, die ihrerseits wieder Probleme anderer Art anregen.

Da es vor Allem darauf anlommen musste, zum Thatbestand der vom Oeffungswinkel abhängigen Wirkungen eine festere Position zu gewinnen, als die mikrographische Literatur geben konnte, habe ich zunächst durch Experimente die Frage zu beantworten gesucht: in welchen Fällen besteht ein Unterschied zu Gunsten des grösseren Oeffnungswinkels und in welchen Füllen besteht ein solcher nicht, wenn alle sonstigen Verschiedenheiten, welche die 
Wirkung möglicherweise beeinflussen können, auf,das Sorgfäligste eliminirt werden? Hierzu hat mir eine Reihe von Objectiven mit sehr verschiedener Brennweite und sehr verschiedener Oeffnung gedient, welche nach meiner Berechnung mit äusserster Accuratesse ausgeführt worlen waren und welche, auf ihre Correctheit noch besonders geprüft," für die Vergleichbarkeit der Beobachtungen sichere Gewähr leisfeten. Als Probeobjecte sind dabei benutzt worden: allerlei Schmetterlingsschuppen und Diatomeenschalen, gestreifte Muskelfasem, Diamanttheilungen auf Glas, Liniensysteme in verschwindend dünnen Silberschichten auf Glas, feinere und gröbere Pulver u. A, daneben aber auch die kleinen optischen Bildchen makroskopischer Objecte (Stabgitter, Drahtgeflecht), welche man durch Luftblasen oder - besser - durch ein auf den Tisch des Mikroslrops gelegtes Objectiv von kurzer Brennweite erhalten kann.

14. Durch solche Versuche hat sich ergeben:

1) So lang'e die freit Oeffunng so gross bleibt, dass aus ihrer Diffractionswirkung keine merkliche Abnahme der Schärfe entspringt, tritt ein Unterschied in der fraglichen Richtung nicht hervor in Bezug auf die Abbildung der Contouren mikroskopischer Objecte, d. h. der Grenzen zwischen ungleich durchsichtigen Theilen im Gesichtsfeld, wofern diese Theile nicht unter ca. 0,01 Mm. herabgehen.

2) Dagegen besteht ein solcher Unterschied constant zu Gunsten des grösseren Oeffnungswinkels gegenüber allen Objecten, welche irgend ein Detail unter jener Grenze der Kleinheit zeigen, gleichgültig, ob dieses Detail durch Unebenheiten der Oberfläche oder durch blosse Unterschiede der Durchsichtigkeit in einer verschwindend dünnen Schicht hervorgerufen wird und gleichgültig, ob es die Form von Streifungen, Gitterzeichnungen, Körnungen etc. hat. Er tritt in gleichem Sinne auch an den erwähnten optischen Bildchen makroskopischer Gegenstände hervor.

3) Je kleinere lineare Dimensionen das betreffende Detail besitzt, desto grösser muss der Oeffnungswinkel des Objectivs sein, wenn jenes bei einer bestimmten Axt der Beleuchtung; z. B. bei rein centraler oder bei möglichst schiefer, wahrgenommen werden soll, und zwar unabhängig von der grosseren odergeringeren Markirtheit der Zeichnung und unabhängig von der Brennweite und der förderlichen Vergrösserung 
des Objectivs. Bei kleinem Oeffnungswinkel kann die Abbildung schon lange vor derjenigen Grenze der Kleinheit, die der förderlichen Vergrösserung entspricht, aufhören.

4) Wo das Detail an wirklichen Objecten in Form von Streifungen, Liniensystemen u. dergl. erscheint, erreicht ein und derselbe Oeffnungswinkel bei schiefem Lichteinfall constant merklich feineres Detail als bei centraler Beleuchtung und zwar ganz unabhängig davon, ob die Beschaffenheit der Objecte die Möglichkeit von Schatteneffecten zulässt oder vollständig ausschliesst.

5) Eine Structur der gedachten Art, welche ein bestimmtes Objectiv bei gerader Beleuchtung nicht wahrnehmbar macht, wird auch nicht wahrnehmbar, wenn man das Object unter beliebigem Winkel gegen die Axe des Mikroskops neigt, selbst wenn dasselbe, senkrecht zur Axe liegend, durch schiefes Licht vollkommen aufgelöst würde. Die Auflösung tritt aber sogleich ein, wenn gleichzeitig der einfallende Lichtkegel senkrecht gegen die Ebene des Objects gerichtet wird. Demnach hängt die Steigerung der Wirkung unter schiefer Beleuchtung ausschliesslich von der Neigung der Strahlen gegen die. Mikroskopaxe, aber nicht von ihrem schiefen Einfall auf das Object ab.

Die hier angeführten Thatsachen belzunden auf der einen Seite die Realität eines von der sonstigen Vollkommenheit der objective und von der Vergrösserungskraft unabhängigen, an den Oeffnungswinkel als solchen geknüpften specifischen Vermögens, und bestimmen dasselbe - in guter Uebereinstimmung mit dem Wortsinne der üblichen Benennung - als ein Vermögen zur Auflösung oder Unterscheidung des mikroskopischen Details; auf der andern, Seite aber enthalten sie auch den unzweideutigen Hinweis darauf, dass die Abbildung sehr feiner körperlicher Structuren auf wesentlich andern Bedingungen als die Abbildung der Contouren gröberer Theile beruhen müsse. In allen Fällen, wo ein derartiges Auflösungsvermögen; d. h. ein directer Einfluss des Oeffnungswinkels, sei es positiv, sei es negativ, in Geltung tritt, kann die dioptrische Wiedervereinigung der von den Objectpunkten ausgehenden Strahlenbüschel in einer Bildfläche unbedingt nicht der zureichende Grund für die Abbildung des Objects sein, weil auf eine solcheVoraussetzung hin die nachgewiesenen Unterschiede absolut unerklärbar bleiben würden. Das Resultat dieser Vorarbeit hat daher die eigentliche Aufgabe 
der Untersuchung dahin formuliren lassen: besondere Ursachen ausserhalb des Mikroskops, welche bei der Abbildung kleinen körperlichen Details mitwirken, nachzuweisen und sodann die Modalitäten ihres Ringreifens in den dioptrischen Vorgang im Einzelnen festzustellen.

Beide Forderungen haben sich. sowohl auf theoretischem wie auch auf experimentellem Wege in einem für die nächsten Bedürfnisse ausreichenden Umfang erledigen lassen.

15. Die Undulationstheorie des Lichts weist in den Erscheinungen der Diffraction oder Beugung eine charaliteristische Veränderung nach, welche körperliche Structuren, nach Maassgabe der Kleinheit ihrer. Dimensionen, an den hindurchtretenden (eventuell auch an reflectirten) Lichtstrahlen hervorbringen. Sie besteht im Allgemeinen in einer Auflösung jedes einfallenden Lichtstrahls in je eine Strahlengruppe von grosser Winkelausbreitung mit periodisch wachsender und abnehmender Intensität innerhalb dieser. Für den besondern Fall von regelmässigen Schichtungen, Streifungen, Punktreihen u. dergl. liefert die mathematische Theorie eine vollstindige Bestimmung der Erscheinung, die alsdann darin aufgeht, dass aus dem einfallenden und geradlinig fortgehenden Lichtstrahl nach entgegengesetzten Seiten hin eine Reihe isolirter Strahlen in regelmässigen Winkelabständen abgesondert (abgebeugt) wird; diese Winkelabstände sind aber für jede einzelne Farbe proportional ihrer Wellenlänge, nehmen also von Violett zu Roth stetig $\mathrm{zu}$, und sind ausserdem umgekehrt proportional der Distanz der Theile in der wirksamen Structur. Wird daher ein milkroskopisches Präparat von der betrachteten Beschaffenheit durch einen Lichtkegel getroffen, wie ihn z. B. der Beleuchtungsspiegel des Mikroskops liefert, so tritt dieser nicht einfach nach seiner geradlinigen Fortsetzung in das Objectiv ein, sondern die Structur des Präparats scheidet aus dem directen Licht eine Anzahl abgelenkter Lichtlsegel mit auseinandertretenden Farben aus, welche je nach der grösseren oder geringeren leinbeit der Structur grössere oder geringere Winkel mit der Richtung der ungebeugten Strahlen bilden. Derartige Objecte senden also Punkt für Punkt m ehrere isolirte Lichtbüschel zum Objectiv, deren Anzahl and Anordnung innerhalb eines bestimmten Winkelraums von der Lage der lichtgebenden Fläche (des Spiegels z. B.) und von der Structur des Präparats abhängt. Diese theoretisch yorauszusagende und auch nach ihren Maass- 
verhältnissen genau zu berechnende Wirkung kann mittelst der unter (4) erwähnten Oeffnungsbilder, welche die Abbildung ler Objecte im Mikroskop begleiten, sehr einfach beobachtet werden. Man stellt ein Object der fraglichen Art mit dem Mikroskop ein, so dass das Detail sichtbar wird, entfernt sodann das Ocular und sieht entweder mit freiem Auge in den offenen Tubus herab oder stellt mit einem passend eingerichteten Hilfsmikroskop von sehr geringer (10-20maliger) Vergrösserung, welches an Stelle des Oculars in den Tubus eingesenkt wird, auf die obere Focalebene des Objectivs ein ${ }^{1}$ ). Man sieht alsdann dits Bild des Spiegels (oder was sonst die lichtgebende Fläche sein mag), wie es von den ungebeugten Strahlen erzeugt wird, ungeben von einer grösseren oder kleineren Anzahl von Nebenbildern, in Form von unreinen Farbenspectren, deren Farbenfolge, vom Hauptbilde aus gerechnet, stets von Blau zu Roth geht.

Objecte mit mehreren sich kreuzenden Liniensystemen zeigen dabei nicht nur in der Richtung der Senkrechten zu jeder Gruppe je eine Reihe von Bengungsbildern, sondern - den Forderungen der Theorie entsprechend - ausserdem noch andere solche Reihen in den Winkeln zwischen jenen Senkrechten. - Schmetterlingsschuppen und Diatomeen zeigen diese Phänomene in der grössten Mannigfaltigkeit. Die gröberen unter ihnen gestatten die. Beobachtung schon mit schwachen Systemen von geringem Oeffnungswinkel; die feineren - von Pleuros. ang. an - verlangen grosse Oeffnung, wenn auch nur die dem Hauptbilde des Spiegels nächstliegenden Beugungsbilder in die Oeffnungsfläche fallen sollen. Für deren Beobachtung ist ein schwaches Immersionsobjectiv am besten geeignet ${ }^{2}$ ).

1) Der allgemeine Charakter dieser Beobachtungsweise ist dadurch gekennzeichnet, dass dabei das abbildende Mikroskopsystem als Objectiv eines Miniatur-Fernrohr's fungirt, indern es die vor ihm liegenden Gegenstände in seinem hintern (obern) Foous abbildet. Der Oeffnungswinkel des Mikroskops wird alsdann das angulare Gesichtsfeld dieses Fernrohrs. Die abbildenden Strahlen müssen dabei sämmtlich das eingestellte Präparat passiren, daher denn alle Ablenkungen, welche dieses bewirkt, in dem kleinen Ferurohrbildchen sichtbaro Nebenbilder der äussern leuchtenden Gegenstände, der Spiegelfläche z. B., herbeiführen. - Es versteht sich von selbst. dass diese $\mathrm{Me}$ thode auch zum Studium der Stractur solcher Präparate dienen kann, in welchen geformte. Theile Ablenkungen dirch Brechung des Lichts herbeiführen.

2) Auf einem ganz andern Wege sind die Beugungsphänomene bei den 
16. Die hier angegebene Methode zur directen Beobachtung der Lichtstrahlung, die von mikroslopischen objecten ausgeht, gèstattet zugleich die experimentelle Entscheidung der Frage, welche Rolle das betrachtete Beugungsphänomen bei der Abbildung der betreffenden Structuren spielt. Die Antwort darauf hat sich ohne Weiteres ergeben, indem ich nach Einstellung der geeigneten Probeobjecte und passender Regulirung des einfallenden Lichts durch Diaphragmen dicht oberhalb dos objectivs, möglichst nahe seinem obern Focus, bald diesen bald jenen Theil der im Beugungsphänomen erscheinenden Strahlengruppe abblendete, um hierauf das mikroskopische Bild des Präparats, wie es von den nicht abgeblendeten Strahlen all ein erzengt wurde, mit dem gewöhnlichen Ocular in Augenschein zu nehmen. - Das Folgende enthält die unmittelbaren Ergebnisse derartiger Experimente; wobei ich nur bemerke, dass alle entscheidenden Versuche mit sehr correcten schwachen Objectiven - 30 bis $6 \mathrm{Mm}$. Brennweite - und den entsprechenden geringen Vergrösserungen ausgeführt sind, stärkere aber, besonders ein Immersionsobjectiv von $3 \mathrm{Mm}$., nur benutzt wurden, um dic an gröberen Objecten schon gewonnenen Resultate an einigen feinen Diatomeen zu controliren. - Die Präparate für die entscheidenden Experimente waren allein Gebilde von genau bekannter Structur: verschiedene, dureh fein zertheiltes Caput mortuum hergestellte Kö̈rnungen, in Glas geritzte Liniensysteme von 0,08 bis $0,002 \mathrm{Mm}$. Linienalstand und ähnliche Liniengruppen in Silberniederschlägen auf Glas, wobei die Silberschicht eine auch für' das stärkste Mikroskop unwahrnehmbare Dicke besass; gekreuzte Liniengruppen ohne Nivenudifferenz wurden durch Uebereinander-

Dirtomeen schon von Flögel (Botan. Zeit. 1869, Nr. 4:3-45) studirt und zur Bestimmung der Streifendistanz benutzt worden. Auch die hier angewandte Beobachtungsmethode kam für letzteren Zweck sehr gut dienen, da der unter (4) angefulurte theoretische Satz es möglich macht, ans dem gemessenen linearen $\Lambda$ bstand dor Bengungsspectra im Oeffnungsbild, unabhängig' von der Richtung dos Lichteinfalls, die Streifendistanz $\mathrm{zu}$ berechnen, wenn man die Brennweite des Objectivs kennt. Ts genügt dazu ein Ocularmikrometer in dem Mikroskop, mit welchem man das Oeffuungsbild beobaohtet. Wemn man zur Beleuchtung eine schmale Lichtfliche unter dem Präparnt und recht intensives Licht verwendet, kann man die Spectra so scharf erhalten, dass bei sehr regelmässigen Streifungen - wie z. B. auf Pleur. ang. - sogar einige Traunhofer'sche Linien sichtibar werden. 
leg'en von zwei einfachen Theilungen, die eine an der untern Fläche des Deckglases, hergestellt. - Die so gewonnenen Thatsachen sind:

1) Wenn alles durch die Beugung aus den einfallenden Strahlen ausgesonderte Licht vollständig abgeblendet wird, so dass allein der übrig bleibende ungebeugte Strahlenbüschel die Abbildung des Präparats vermittelt, so hat diess auf die Schärfe der Contouren zwischen ungleich durchsichtigen Stellen des Sehfeldes keinen Einfluss, so lange das Diaphragma gross genug bleibt, um nicht durch seine Diffractionswirkung eine sichtbare Depression der förderlichen Vergrösserung herbeizuführen. Auch wird dadurch die deutliche Wahinehmung getrennter 'Theile nicht in merklichem Grade behindert, wenn deren nicht mehr als 30-50 auf den Millim. kommen ${ }^{1}$ ). Je weiter aber diese Zahl überschritten wird, desto mehr verschwimmt das Detail, so dass schon bei ca. 100 pro Mm. nur eine gleichförmige Fläche sichtbar bleibt, welche Vergrösserung auch angewandt wird, und zwar ebenso bei gerader wie bei beliebig schiefer Beleuchtung. Schon zwei Theile, z. B. zwei Diamantstriche oder zwei Linien in einer Silberschicht, werden unter den bezeichneten Verhältnissen ununterscheidbar und erscheinen als ein breiterer Strich mit schar fen Contouren. Mit dem stäriksten Immersionsobjectiv ist von der Zeichnung auf Pleur. ang. nicht das geringste zu erkennen; und die groben Längsstreifen auf Hipparchia Jan. bleiben selbst bei 200facher Vergrösserung unwahrnehmbar. - Bei Körnungen und anderem unregelmässigen Detail lässt sich das gebeugte Licht von dem ungebeugten nicht vollştändig sondern. Dem entsprechend tritt bei möglichster Abblendung zwar kein absolutes Verschwinden aller. Theile, aber doch eine so grosse Undeutlichkeit des Bildes ein, dass der feinere Inhalt des Präparats in gleichmässiges Grau übergeht.

2) Wenn alle Strahlen abgeblendet werden bis auf einen du'ch Beugung erzeugten Büschel, so liefert dieser ein mehr oder minder lichtstarkes positives Bild von den die Beugung bewirkenden Theilen des Objects in dunklem Felde, aber gleichfalls obne alles Detail. (Theilungen erscheinen als gleichmässig helle Flächeustreifen auf schwarzem Grunde.)

1) Diese Grenzbestimmung ist desshalb unsicher, weil bei geringer Ablenkung der gebeugten Strahlen nur durch sehr enge Diaphragmen die vollständige Abblendung zu erreichen ist. 
3) Wenn dagegen mindestens $z$ wei getrennte Lichtbüschel in das Mikroskop eingelassen werden, so zeigt das Bild stets ein scharf marlkirtes Detail, sei es in Form eines oder mehrerer Liniensysteme oder in Form isolirter Felder. Dabei ist es gleichgiltig, ob unter den eintretenden Strahlenkegeln das ungebeugte Licht enthalten ist oder nicht, d. h. ob das Bild in hellem oder in dunklem Gesichtsfeld erscheint. Andere Lichtbüschel in Wirksamkeit gesetzt, entsteht aber immer anderes Detail-verschieden, entweder nach dem Grade der Feinheit oder nach der Art der Zeichnung - ; und dieses Detail braucht weder dem Inhalte des mikroskopischen Bildes bei normaler Beleuchtung noch auch der anderweit bekannten wirklichen Structur des Objects conform zu sein. Einige nähere Bestimmungen in Betreff des letzten Punktes enthalten die beiden folgenden Sätze.

4) Ein einfaches Streifensystem wird zwar stets auch als solches abgebildet, wenn zwei oder mehrere Lichtbüschel zur Wirkung gelangen, aber in doppelter, dreifacher ... Feinheit, sobald unter jenen nicht zwei co nsecu tive Büschel enthalten sind, sondern ein, zwei, . . . zwischenliegende übersprungen werden; eine Gruppe von nur zwei Linien im Object z. B. erscheint dabei als aus drei, vier . . getrennten Strichen zusammengesetzt. Die so erzengten Scheinbilder sind aber in Hinsicht auf ihre Schärfe und die Constanz ihres Auftretens bei keiner Vergrösserung von dem normalen Bilde einer wirklich doppelt, dreifach, ... so feinen Streifung gleicher Art zu unterscheiden, wie sich durch ein eclatantes Experiment, bei welchem eine solche verdoppelte Theilung unmittelbar neben dem Bilde einer wirklich doppelt so feinen im Gesichtsfeld erscheint, darthun lässt.

5) Wenn zwei einfache Gitter in demselben Niveau unter beliebigem Winkel sich kreuzen, so kann man nicht nur durch geeignete Regulirung des Lichtzutritts nach Belieben beide Liniensysteme einzeln oder gleichzeitig sichtbar machen, sondern man kann auch durch andere Formen der Abblendung in gleicher Schärfe und Markirtheit zahlreiche neue Liniensysteme, welche als solche im Object gar nicht vorhanden sind, und mannigfach geformte Felder, zur Erscheinung bringen. Die neu aufretenden Liniengruppen entsprechen dabei, nach ihrer Lage und der Liniendistanz in 
ihnen, stets den möglichen Formen, nach welchen sich die Kreuzungsunkte, der wirklichen Striche im Object in äquidistante Reihen ordnen lassen.

So zeigt $u$. A. ein Kreuzgitter mit senkrechter Kreuzung zwei secundäre Streifungen in den Richtungen der Diagonalen, deren Linienabstand zur Distanz der wirklichen Striche sich wie $1: \sqrt{2}$ verhält; aber auch noch vier (zarter gezeichnete) im Verhäitniss von $1: \sqrt{5}$ engere Gruppen, welche je um ca. $27^{\circ} \mathrm{zu}$ einer Gitterrichtung. genejgt sind. Bei einer Kreuzung unter $60^{\circ}$ tritt, abgesehen von anderen feineren Liniensystemen, in gleicher Stärke mit den im Object wirlklich vorhandenen ein drittes System mit demselben Linienabstand auf, um $60^{\circ}$ Grad gegen die andern geneigt, und man erblickt, wenn alle drei gleichzeitig erzengt werden, statt der rhombischen Felder vollkommen scharf begrenzte Sechsecke, ganz von der Art wie auf Pleur. ang. zu sehen sind. - Ich füge hinzu, dass alle hier namhaft gemachten, dex Structur der bekannten Objecte nicht conformen Erscheinungen stets bei derselben Finstellung, welche auch das normale Bild scharf hervortreten lässt, beobachtet werden und dass sie bei den verschiedensten Combinationen von Objectiven und Ocularen, wenn die Beleuchtung in derselben Weise regulirt wird, mit derselben Constanz eintreten. Der Einfuss der Diffraction, welche die Biaphragmen über dem Objectiv ausiiben, ist durch directe Controlversuche eliminirt worden.

Weiteres Detail über diese Phänomene kann nicht gegeben werden ohne genaueres Eingehen auf die theoretischen Gesetze der Diffraction: Aus dem gleichen Grunde unterlasse ich hier, auch den Zusammenhang zwischen den eben angefürten Thatsachen und dem unter (14) erwälmten Verhalten kleiner optischer Bildchen nachzuweisen. Dagegen sei noch das Folgende hervorgehoben:

6) Die in den beschriebenen Experimenten benutzte Manipulation, das partielle Abblenden der vom Object ausgehenden Lichtstrahlung, kommt unter den gewöhnlichen Verhältnissen bei der Beobachtung aller feineren mikroskopischen Gebilde unbeabsichtigter aber auch unvermeidlicher Weise zur Anwendung. Denn sobald das Detail eines Objects in seinen linearen Dimensionen auf $k$ leine Vielfache von der Wellenlänge des Lichts ${ }^{1}$ ) herabgeht, kann - wic

1) Die Wellenlünge des Roth ist $=0,76 \mu$; des dunkeln Blau $=0,43 \mu$. Zur Vergleichung sei der Streifenabstand auf einigen bekannten Probeobjecten 
die Rechnung und die Beobachtung zeigt - auch ein sehr grosser Oeffnungswinkel des Objectivs nie mehr als einen kleinen Theil der ganzen durch die Beugung erzeugten Strahlengruppe gleichzeitig aufnehmen. Dieser Theil aber wird immer ein anderer, sowohl wenn bei gleich bleibender Beleuchtungsrichtung der Oeffnungswinkel grösser oder kleiner wird, wie auch wenn bei constantem Oefinungswinkel die Beleuchtung wechselt. Hierauf kommen alle - mir bekannten - Modificationen zurück, welche die Bilder feiner Structuren unter anderem Oeffungswinkel und unter anilerem lichteinfall bei aberrationsfreien Objectiven zeigen. Die constante Steigerung des Auflösungsvermögens durch schiefe Beleuchtung namentlich, also sowohl das Sichtbarwerden von neuem Detail, wie auch das marlirtere Hervortreten des bei centralem Licht schon erkennbaren, ist in allen Fällen allein dadurch bedingt, dass bei schiefem Lichteinfall Beugungsbüschel in die freie Oeffnung hereintreten, die wegen ihrer starlien Ablenkung sonst ausserhalb derselben bleiben, oder dass Beugungsbinschel, die bei centraler Beleuchtung nur unvollkommen aufgenommen werden, jetzt voll in das Mikroskop eintreten und, unter gleichzeitiger Abschwächung der ungebeugten Strahlen, mit grösserer Intensität zur Wirkung gelangen. - Abgesehen hiervon aber kommen bei der gewöhnlichen Beobachtungsweise auch solche Abblendungsformen vor, welche die Bedingungen für die unter (5) aufgefühten Wirkmngen euthaiten. In Folge dessen können an allen Objecten, welche überhaupt $z$ wei einigermaassen gleichartige Streifungen darbieten, durch blosse Veränderung des Lichteinfalls deren noch mehrere in andern Richtungen sichtbar gemacht werden, wofern der Oeffnungswinkel des angewandten Objectivs zur Feinheit der Streifung ein passendes Verhältniss hat - wie $u$. A. an mehreren Diatomeen deutlich hervortritt. Selbst Abblendungen von solcher Wirkung wie in den Experimenten unter (4) können vielfach unbeabsichtigter Weise eintreten; es erklärt sich daraus z. B. das Auftreten feiner Längslinien zwischen den groben Läugsstreifen auf Hipp. Jan., wie starke Objective unter gewissen Spiegelstellungen solche sichtbar machen.

T. Die hier aufgeführten Thatsachen scheinen mir hinreichend, um theils unmittelbar, theils in Verbindung mit unanfechtbaren

hier angeführt: Längsstroifen anf Hipp. Jan. $2 \mu$, Querstreifon $0,7 \mu ;$ Pleuros. ang. $0,48 \mu$; Surirella Gemma $0,3 \mu$; Frustulia Saxonica $0,25 \mu$. 
Lehrsätzen der Undulationstheorie, eine Reihe wichtiger Schlussfolgerungen, welche sowohl die milkroskopische Wahrnehmung als solche, wie auch die Einrichtung und den Gebrauch des Mikroskops berühren, sicher zu begründen. - Zunächst die Consequenzen in der ersten Richtung:

Solche Theile der mikroskopischen Präparate, welche entweder durch ihre Isolirtheit (einzelne Fäden, Körnchen u. dergl.) oder durch ihre, relativ zu den Lichtwellen, beträchtliche Grösse, selbst keine Beugung von merklichem Betrage herbeiführen, bilden sich im Mikroskop nach den gewöhnlichen dioptrischen Gesetzen der Strahlenconcentration $a b$. Die Abbildung geschieht dabei rein negativ, nämlich allein durch den ungleichen Lichtausfall, den in homogenen Massen partielle Absorption (z. B. Färbung) oder theilweise Ablenkung der Strahlen durch Brechung, in Theilen mit innerer Structur aber die durch diese bedingte Bengung hervorbringt, Das so erzeugte Absorptionsbild ist dem Object selbst unbedingt ähnlich und lässt bei richtiger stereometrischer Deutung desflächenhaft Gesehenen einen vollkommen sichern Rückschluss auf die - morphologische - Zusammensetzung desselben zu.

Alle feinere Structur hingegen, deren Elemente klein und nahe genug sind, um durch ihr Nebeneinandersein ein merkliches Beugungsphänomen hervorzurufen, wird nicht more geometrico abgebildet, d. h. nicht so, als ob die vom Object ausgehenden homofocalen Strahlenbüschel, indem sie nach vielerlei Veränderungen schliesslich wieder homofocale Büschel mit reellen oder virtuellen Vereinigungscentren werden, .jenes Punkt für Punkt auf einer Bildfläche copirten. Denn wenn auch alle dioptrischen Bedingungen hierzu vollständig erfüllt sind, enthält doch das Bild Nichts von solchen Elementen, wofern nicht mindestens zwei der Theilstrahlen, welche durch die Zerlegung des Lichts entstehen, wieder vereinigt werden.

Für Jeden, der sich die Voraussetzungen deutlich macht, auf denen die gewohnte Annahme der Aehnlichkeit zwischen einem Objecte und seinem optischen Bilde beruht, muss das Gesagte zu dem Schlusse ausreichen: dass unter den nachgewiesenen Umständen eine solche Annahme eine vollkommen willkürliche Hypothese wird. Als positive Instanz steht ihr aber die Folgerung entgegen, zu welcher die unter 4) und 5) angeführten Versuche, bei genauerer 
Discussion ihrer Resultate, führen: dass verschiedene Structuren stets das nämliche mikroskopische Bild liefern, sobald die Verschiedenheit des an sie gein nupften Beugungseffectes für das Mikroskop künstlich beseitigt wird; und dass gleiche Structuren stets verschiedene Bilder liefern, wenn der Beugungseffect, in dem für das Mikroskop wirksam werdenden Theil, küntlich ungleich wird. Damit ist aber gesagt, dass die unter Mitwirkung des Beugungsvorgangs entstandenen Structurbilder in keinem constanten Zusammenhang mit der wirklichen Beschaffenheit der sie veranlassenden objecte, vielmehr blos in constantem Zusammenhang mit dem die Abbildung vermittelnden Diffractionsphänomen stehen.

$\mathrm{Es}_{\mathrm{s}}$ ist hier nicht der Ort, auf die physikalische Erklärung dieser Erscheinungen näher einzugehen. Doch sei angefithrt, dass die hier aus den unmittelbar beobachteten Thatsachen gezogenen Schlüsse in der Undulationstheorie des Lichtes ihre vollständige Rechtfertigung finden. Nicht nur lässt sich nach deren Grundsätzen davon Rechenschaft geben, warum mikroskopisches Structurdetail nicht nach dioptrischen Regeln abgebillet wird, sondern es lässt sich auch genau nachweisen, wie die anderweitige Art von Abbildung, welche die Beugungsbüschel hervorrufen, zu Stande kommt. Man kann zeigen, dass die in der obern Focalebene des Objectivs auftretenden Bilder der lichtgebenten Fläche - das directe Bild und die durch Beugung entstandenen Nebenbilder - in correspondirenden Stellen je gleiche Schwingungsphasen repräsentiren müssen, wenn man jede einzelne Farbe für sich betrachtet. Diese Oeffnungsbilder verhalten sich demnach ganz ebenso, wie z. B. die beiden Spiegelbilder einer Lichtflamme beim Fresnel'schen Interferenzversuch; das Zusammentreffen der von ihnen ausgehenden Strahlen muss in Folge der eintretenden Interferenzen einen periodischen Wechsel von Licht und Dunkelheit herbeiführen, dessen Form- und Maassverhältnisse von der Zahl, der Anordnung und dem gegenseitigen Abstand der interferirenden Lichttächen abhängen. Die im Gesichtsfeld des Mikroskops erscheinende Structurzeichnung ist in allen ihren Merkmalen, sowohl in denjenigen, die der Beschaffenheit des Objects möglicher Weise conform, wie auch in denjenigen, die ihrnicht conform sind, nichts Anderes als das Resultat des Interferenzvorgangs beim 
Zusammentreffen der sämmtlichen wirksamen Strahlenbüschel.

Der unter (4) erwähnte Satz ïber den Zusammenhang zwischen len linearen Abständen im Oefhungsbild und den Richtungsdifferenzen der eintretenden Strahlen, in Verbindung mit der in (6) gelehrten schematischen Zerlegung des Mikroskops, gewährt die ausreichende Grundlage zur vollständigen Durchführung dieser Folgerung. Es lässt sich daraus ableiten, dass bei einem achromatischen $\mathrm{Ob}-$ jective die Interferenzbilder für alle Farben coincidiren, also - zum Unterschied von allen sonst bekannten Interferenzphänomenen -. eine achromatische Gesammtwirkung ergeben müssen; ferner, dass die Massverhältnisse der so erzeugten Bilder von denen der wirklichen Structur stets so abhängen, wie die lineare Vergrösserung des Mikroskops fiur dioptrische Bilder es mit sich bringen würde, und zwar bei jeder Einrichtung des optischen Systems und (von möglichen Verschiedenheiten des Beugungseffectes abgesehen) bei jeder Art der Beleuchtung; u. A. m. Nicht nur finden daraufhin alle 'Thatsachen der Beobachtung, wie sie unter (16) angeführt sind, ihre genügende Erklärung; cs lässt sich auch das Structurbild, welches irgend ein beliebiges Object bei einer bestimmtèn Beleuchtung zeigen wird, in allen Einzelheiten vorausberechnen, wenn man nur das zur Wirkung kommende Beugungsphänomen nach Zahl, Anordnung und Lichtstärke der sämmtlichen Beugungsspectragegeben erhält.

18. Das Endresultat dieser Betrachtung stellt sich demnach dahin :

Alle diejenigen Erscheinungen im mikroskopischen Bild, welche nicht schon mit dem blossen Absorptionsbilde gegeben sind, sondern der Mitwirkung der durch Beugung entstandenen Strahlengruppen bedürfen, d. h. alle Anzeigen von Structurdetail, liefern im Allgemeinen keine der wirklichen Beschaffenheit der Objecte conforme, d. h. geometrisch ähnliche, Abbildung. Wi e constant, markirt und scheinbar körperlich derartige Anzeigen (Streifensysteme, Felderzeichnungen u. dergl.) im Mikroskop auch auftreten mögen, so dürfen sie doch nicht morphologisch, d. h. als Bilder körperlicher Formen, sondern nur physikalisch, d. h. als Merkmale nicht als Abbilder - gewisser materieller Verschiedenheiten in oder an den betreffenden 'Theilen gedeutet werden; und $\mathrm{zwar}$ kann aus dem mikroskopischen Befund mit Sicherheit auf 
Nichts weiter geschlossen werden, als auf das Vorhandensein solcher Structurbedingungen, als zur Erzeugung des die Abbildung vermittelnden Beugungsphänomens nothwendig und hinreichend sind.

Je kleiner die linearen Maasse einer Structur werden, eine desto geringere Anzahl von Beugungsbüscheln kann auch beim grössten Oeffnungswinkel effectiv werden; desto weniger kann die Intensitätsahstufung in der Reihe dieser Büschel solcho Verschiedenheiten, die innerhalb derselben Maassverhältnisse noch möglich sind, zum Ausaruck bringen; desto weniger bes ti $m \mathrm{~m} t$ wird Dasjenige, was von der wirklichen Strutur aus dem Bilde (oder auch aus dem sichtbaren Beugungsphänomen) rückwärts erschlossen werden kann.

Von diesem Staudpunkte aus erscheinen u. A. alle Versuche, den Bau der feineren Diatomeenschalen durch morphologische Deutung ihrer mikroskopischen Bilder festzustellen, als auf unzulässige Prämissen gegründet. $O \mathrm{~b}$ z. B. Pleur, angulatum zwei oder drei Streifensysteme besitze; ob überhaupt wirkliche Streifung vorliege, oder ob die sichtbaren Zeichnungen von isolirten Erhöhungen, oder von isolirten Vertiefungen herrihren u. dergl, darüber kann kein noch so vollkommenes Mikroskop und keine noch so hohe Vergrösserung Aufschluss geben. Was sich behaupten lässt ist allein das Dasein der optisch nothwendigen Bedingungen fur den die Abbildung begleitenden Betugungseffect. Sowoit dieser aber irgend einem Mikroskop jemals zugïnglich sein kann - sechs symmetriseh liegende Spectra, bei normalem Lichteinfall um ca. $65^{\circ}$ (für Blau) gegen die Richtung der ungebeugten Strahlen abgelenkt - kann er von jeder beliebigen Structur herrühren, welche optisch heterogene Elemente (im Imern oder an der Oberflïche) in irgend einer Art nach einem System gleichseitiger Dreiecke von $0,48 \mu$ Höhe georduet enthält. Was diese Elemente sein mögen, geformte Theile oder rein moleculare Texturdifferenzen (Verdichtungscentra), immer werden sie die bekannteu sichtbaren Zeichnungen ergeben. Es fällt jeder Grund fort, die fraglichen Structurelemente als gestaltet (als Erhöhungen oder Vertiefungen) vorauszusetzen, nach dem erwiesen ist, dass weder das Sichtbarwerden der betreffenden Zeichnung überlaupt noch ihr stärkeres Hervortreten bei schiefer Beleuchtung mit Schatteneflecten irgend etwas zu thun hat ${ }^{1}$ ). Die Vertheilung ron

1) Die Veränderungeu, wolche das Bild von Pl. ang. boim Huben und 
Hell und Dunkel auf der Fläche der Schale in Form eines Systems von sechseckigen Feldern erscheint als das mathematisch nothwendige Resultat der Interferenz zwischen den sieben isolirten Lichtbüschem, welche die Bengung erzeugt, was deren physischer Grund in Object auch sein mag; die Lage der Sechsecke - zwei Seiten parallel der Mittelrippe - hat ihren zureichenden Grund in der sichtbaren Orientirung der Beugungsspectra gegen die Axe der Schale und kann daraus 'durch Rechnung abgeleitet werden; ohne dass man über den wirklichen Bau des Objects irgend etwas zu wissen braucht.

Dass auch für eine grosse Zahl rein organischer Gebilde auf dem Arbeitsfeld der Histologen im Wesentlichen die nämliche Sachlage besteht, kann das Beispiel der gestreiften Muskelfaser lehren. An guten Präparaten derselben lassen sich die Beugungserscheinungen ziemlich leicht beobachten und ihre Wirkungen im mikroskopischen Bild auf dem früher beschriebenen Wege experimentell studiren. Die mannigfachen Veränderungen in den Charakteren des Bildes, die sich dabei ergeben, erläutern einigermaassen den bekanaten ' $Z$ wiespalt zwischen den Befunden verschiedener Beobachter in Bezug auf diese Gebilde, bekunden aber auch die Unmöglichkeit, über ihre wirkliche körperliche Zusammensetzung im Sinne der bisherigen Bemühungen irgend etwas Haltbares auszumachen.

Was hier bezüglich der Grundlagen mikroskopischer Wahrnehmung geltend gemacht worden ist, trifft übrigens nicht allein die morphologischen Verhältnisse der Objecte, sondern ebenso sehr die sonstigen Eigenschaften, welche aus der mikroskopischen Beobachtung erschlossen werden, sollen. Dass Unterschiede der Durchsichtigkeit und Färbungen, die man im Bilde wahrnimmt, nicht nothwendig: Merkmale der Objecte zu sein brauchen, sondern oft aus der gänzlichen oder partiellen Abblendung von Beugungsbüscheln entspringen können, wird durch bekannte Erscheinungen an den Diatomeen hinlänglich illustrirt. Wichtig aber scheint es, darauf hinzuweisen, dass

Senken des Tubus erfährt, können äber das Vorhandensein von Erhöhungen schon desshalb nichto beweisen, weil sie bei gekreuzten Diamantheilungen ganz in gleicher Weise auftreten. Ausserdem lässt die Beobachtung einer scharf begrenzten Lichtquelle durch eine P1. Schale hindurch - nach dex wiederholt erwähnten Methode - an den sie passirenden Lichtstrahlen keiner* lei Ablenkung durch Brochung erkennen; die Schale verbält sich in dieser Hinsicht genau wie eine parallelfächige Glasplatte. 
auch Polarisationsmerkmale an den Bildern von Objecten mit mikro* skopischer Structur in mehrfacher Hinsicht anders beurtheilt werden müssen wie solche an reinen Absorptionsbildern. Von ihnen ohne Weiteres auf doppeltbrechende Substanzen, im gewöhnlichen Sinne, zu schliessen, ist mindestens sehr misslich. Denn es bleibt die Möglichkeit offen, dass dieselben Texturbedingungen, welche die Beugung bewirken, unter Umständen auch gleichzeitig Polarisationseffecte hervorrufen, die, als eine blosse Function des Beugungsphänomens, gar nicht wie bei Krystallen, Stärkekörnern u. dergl. vom Durchtritt der Lichtstrahlen an sich abhängen. Dass Derartiges in der That vorkommt, wird mir durch einige Wahrnehmungen an Pleur. ang. und anderen Diatomeenskeletten wahrscheinlich gemacht. Wenigstens zeigen* die Beugungsbüschel bei mehreren Objecten dieser Art, mit polarisirtem Licht beobachtet, Modificationen, welche schwer auf andere Weise zu erklüren sein dürften. - Wie dem aber auch sein mag, jedenfalls bleibt es unzulässig, bei einem Object wie die gestreifte Muskelfaser z. B., dessen Detail nicht dioptrisch abgebildet ist, aus den Veränderungen des Beugungsbildes im polarisirten Licht nach den gewöhnlichen Kriterien auf nebeneinanderliegende einfach - und doppeltbrechende Schichten zu schliessen. Denn wenn eine gleichmässig doppeltbrechende Substanz mit irgend einer zur Erzeugung des bestehenden Beugungseffectes ausreichenden Differenzirung vorläge, so würde die aus der Interferenz der polarisirten Beugungsbüschel entspringende Streifung ganz ebensolehe Modificationen zeigen mitssen, wie die Muskelfaser im polarisirten Licht erkennen lässt.

19. In Anschluss an die vorstehenden, für den wissenschaftlichen Gebrauch des Mikroskops wichtigen Schlussfolgerungen orgeben sich ferner ganz bestimmte Grenzen für das Unterscheidungsvermögen sowohl jedes einzelnen Objectivs, wie auch des Mikroskops überhaupt.

Durch kein Mikroskop können Theile getrennt (oder die Merkmale einer real vorhandenen Structur wahrgenommen)werden, wenn dieselben ein ander so nahe stehen, dass auch der erste durch Beugung erzeugte Lichtbüschel nicht mehr gleichzeitig mit dem ungebeugten Lichtkegel in das Objectiv eintreten kann. Daraus entspringt für jede Grösse des Oeffnungswinkels eine bestimmte kleinste Distanz des Unterscheidbaren, deren numerische Angabe nur desshalb un- 
sicher bleibt, weil sie, der ungleichen Wellenlänge wegen, für Licht der verschiedenen Farben ungleich ist, das relative Gewicht der einzelnen Farben aber bei der Beobachtung sehr wechselt. Irgend eine bestimmte Farbe zu Grunte gelegt, ergiebt sich der betreffende Ninimalwerth für $x$ ein centrale Beleuchtung durch Division der Wellenlänge mit dem Sinus des halben Oeffnungswinkels, für den höchsten zulässigen Grad schiefer Beleuchtung aber bei jedem Oeffnungswinkel genau halb so gross - sonst gleiche Umstände vorausgesetzt. - Da nun auch beim Immersionssystem der Oeffnungswinkel durch kein Mittel erheblich über diejenige Grösse, die $180^{\circ}$ in Luft entsprechen würde, hinausgeführt werden kann, so folgt, dass, wie auch das Mikroskop in Bezug auf die förderliche Vergrässerung noch weiter vervollkommnet werden möchte, die Unterscheidungsgrenze für centrale Beleuchtung doch niemals über den Betrag der ganzen, und für äusserste schiefe Beleuchtung niemals über den der halben Wellenlänge des blauen Lichts um ein Nennenswerthes hinausgehen wird.

Letztere Grenze ist - für das directe Sehen - bei dèn feinsten bekannten Diatomeenstreifungen und bei den letzten Liniengruppen der Nobert'schen Platte thatsächlich schon erreicht. Nur bei photographischer Aufnahme der mikroskopischen Bilder kann die Unterscheidung noch merklich weiter reichen. Denn wegen der bedeutend kürzeren Wellenlänge der chemisch wirksamen Strahlen werden bei jedem Objectiv die Bedingungen für die photographische Abbildung sehr viel günstiger; nämlich so, wie sie für das directe Sehen eine im Verbältniss von $3: 2$ gröbere Structur stellen würde ${ }^{1}$ ).

\section{Das optische Vermögen des Mikroskops.}

29. Die vorstehenden Erörterungen gewähren die Grundlage fuir eine sichere Feststellung der Functionen, welche das optische Vermögen des Mikroskops ausmachen und damit zugleich für eine rationelle Formulirung der Anspriiche, welche an die optische Einrichtung desselben $\mathrm{zu}$ stellen sind.

1) Hieraus ist - von andern Gründen ganz abgesehen - zu entuehmen, dass die Leistung eines Objectivs bei photographischen Aufnahmen Keinen Maasstab für seine Leistung im gewöhnlichen Gebrauch abgeben kann. 
Die seit lange in der mikrographischen Literatur eingebürgerte Unterscheidung eines Definitions- und eines Auflösungsvermögens gewinnt durch die gegebenen Nachweise eine sehr viel grössere Tragweite, als ihr den bisher bekannten 'Thatsachen gegenüber füglich hat beigelegt werden kömnen. Diesen Nachweisen zufolge ist - von zwei nahe liegenden Ausnahmefällen abgesehen - das mikroskopische Bild im Allgemeinen eine Superposition ' $z$ we i er Bilder von ganz verschiedenem Ursprung und ginz verschiedener Natur, die sich auch thatsächlich von einander tremnen lassen - wie die im vorigen Abschnitt beschriebenen Exporimente zeigen. Das eine ist ein negatives Bild, in welchem sich die 'Theile des Objects vermöge des ungleichen Lichtausfalls; den ihre Masse an den hindurchtretenden Strahlen bewirkt, geometrisch älmlich abbilden. Mau kann es kur das $\Lambda$ bsorptionsbild nennen, weil partialle Absorption die hauptsächliehste Ursache des Lichtausfalls darstellt. Fs ist der Träger des Definitionsvermögens, dessen Höhe, nach den Bedingungen dieser: Art Abbillung, allein durch die grössere oder geringere Vollkommenheit bestimmt ist, mit welcher der direct einfallende Lichtikegel zu homofocaler Vereinisung gebracht wird. Demnach ist es immer dieser directe Lichtkegel, wie er durch Lage und Ausdehnung der lichtgebenden Fläche gegeben ist, welcher definirt, gleichgiltig in welcher Richtung er in das Objectiv gelangt, d. h. gleichgiltig, ob ein centraler oder ein peripherischer 'Theil der freien Oeffnung ihn aufnimnt. Unabhängig aber von diesem Absorptionsbilde werden solche 'Theile des Objects, wolche imsere Structur enthalten, nochmals wiedergegeben, und zwar in einem positiven Bilde, weil dieselben in Folge des eintretenden Beugungsphänomens quasi selbstleuchend werden. Dieses zweite Bild, welches man das Beugungsbild nennen kann, besteht zwar selbst streng genommen ans so viel partiellen Bildern, als aus dem einfallenden Strahlenkegel isolirte Lichtbüschel abgesondert werden und in das Objectiv eintreten, indem, den angefübrten Experimenten zufolge, jeder einzelne von diesen schon ein positives Bild erzengt; weil aber diese partiellen Bilder, einzeln genommen, inhaltleer sind, das sichtbare Detail erst durch die Verschmelzung mehrerer erzeugt wird, kommt praktisch nur der Gesammteffect aller als selbständiger Factor in Betracht. - Dieses resultirende Beugungsbild erscheint nun offenbar als der Träger des Aufösungs- oder Unterseheidungsvermögens des Milkroskops. Seine Entwickelung hïngt demmach zunächst und in erster 
Reihe yom Oeffnungswinkel ab, insofern dieser allein - nach oben angegebenen Normen - die Grenze seiner möglichen Leistung bestimmt; seiner thatsächlichen Höhe nach aber hängt es zugleich ab von der Vollkommenheit, in welcher die partiellen, den einzelnen Beugungsbüscheln entsprechenden Bilder zur Verschmelzung gelangen; denn letztere erst ruft das Detail hervor, welches die Merlxmale oder Anzeigen bestimmter Structurthatsachen liefert. Da nun aber die einzelnen Strahlenbüschel, deren confocale Vereinigung hiernach gefordert wird, verschiedene und je nach der Structur des. Objects und der Beleuchtungsart immer wechselnde Theile der freien Oeffnung in Anspruch nehmen - wie man nach der mehrfach benutzten Beobachtungsweise, meist schon durch einen Blick in den offenen Tulus des Mikroskops, constatiren kann - so ist eine in allen Fällen gleich vollkommene Verschmelzung der verschiedenen Elemente des Beugungsbildes unter sich und nächstdem eine correcte Superposition desselben auf das neben ihm erzeugte Absorptionsbild offenbar nur dann möglich; wenn das Objectiv für den ganzen Umfang seiner freien Oeffnung gleichmässig frei von sphärischer Aberration ist.

21. Nach den bisher geltenden Vorstellungen über den Vorgang der Abbildung im Mikroskop durfte man annehmen, dass Aberrationsreste in den Objectiven nur die Schärfe der Abbildung beeinträchtigen würden, and dass solche demnach als nicht vorhanden oder doch als praktisch irrelevant anzusehen seien, soweit kein sichtbarer Mangel jener Art vorhanden ist.

Die hier nachgewiesenen Umstände, zusammengehalten mit Demjenigen, was unter (7) über die typische Form der sphärischen Aberration bei grossem Oeffnungswinkel gesagt ist, stellen deren Bedeutung in ein wesentlich anderes Licht. Die einzelnen Elemente des mikroskopischen Bildes, sowohl das Absorptionsbild wie die verschiedenen Bestandtheile des Beugungsbildes, werden durchweg durch isolirte Lichtkegel von relativ geringem Divergenzwinkel - fast nie über $30-40^{\circ}$ - erzeugt. Auch bei einem ansehnlichen Rest sphärischer Aberration künnen die Spitzen solcher isolirter Büschel, jedes für sich betrachtet, scharf genug sein, um keinen merklichen Zerstreuungskreis übrig zu lassen. Da aber bei grossem Oeffnungswinkel diese einzelnen Büschel die verschiedensten Theile der freien Oeffunng gleichzeitig in Thätigkeit setzen, so können im vorausgesetzten Falle ihre Spitzen nicht in ein em Punkte zusammentreffen, 
sondern müssen hinter und nebeneinander auftreten. Die Bestandtheile des Gesammtbildes kommen daher nicht zu correcter Verschmelzung, sondern werden vielmehr longitudinal und lateral gegeneinander verschoben. Die ein und derselben Stelle und ein und demselben Nivenu des Objects zugehörigen Structurmerkmale, wie z. B. verschiedene Streifensysteme, erscheinen daher sowohl von einander getrennt, wie auch getrennt von den Contouren der Objecttheile, zu denen sie gehören. - In Folge der Einseitigkeit, mit welcher in neuerer Zeit die Vervollkommnung des Mikroskops auf die Vergrösserung des Oeffnungswinkels gerichtet gewesen ist, sind die Bedingungen für derartige Abnormitäten, zumal für trïgerische Niveaudifferenzen, bei neueren starken Objectiven zum Theil in der ausgiebigsten Weise vorhanden - wie zahlreiche Erfahrungen mich belehrt haben; und ich bin sicher, nicht fehlzugreifen, wenn ich die Meinung ausspreche, dass die Consequenzen dieser Sachlage in vielen Streitfragen der Mikrolkospiker über verwickelte Structurverhältnisse eine unerwartet grosse Rolle spielen.

Da Jeder als die oberste Anforderung, welche die Rücksicht auf den wissenschaftlichen Gebrauch an das Mikroskop stellt, die anerkennen wird, dass Zusammengehöriges im Object auch im Bild als Zusammengehöriges dargestellt werde, und zwar bei jeder Structur und bei jeder Art der Beleuchtung, so folgt, dass die gleichmässige Correction der sphärischen Abweichung für den ganzen Unfang der freien Oeftnung die entscheidende Richtschnur bei der Construction der Mikroskope sein muss. Nun zeigt sich, wie unter (7) angeführt, dass beim Trockensystem eine gehörige Ausgleichung der sphär. Aberration über einen Oeffnungswinkel von $110^{\circ}$ hinaus thatsächlich unmöglich wird; man gelangt daher zu dem Schlusse, dass ein Trockenobjectiv für den normalen wissenschaftlichen Gebrauch um so weniger geeignet sein kann, je feinere Streifensysteme, über die jenem Oeffnung'swinkel entsprechende Unterscheirlungsgrenze hinaus - ca. $0,35 \mu$ für schiefes Licht - es noch sichtbar macht. - Die möglichste Steigerung des Auflösungsvermögens kann rationeller Weise nur beim Immersionsobjective als Ziel gesteckt werden, da allein die Immersion die Möglichkeit gewährt, ohne mit jener obersten Anforderung in Widerspruch zu kommen, den Oeffnungswinkel beliebig (d. $h$. bis an die Grenze des technisch Ausführbaren) $\mathrm{zu}$ vergrössern ${ }^{1}$.

1) Die in dem nenen Catalog von C. $Z_{0}$ is in Jena aufgeführten Mi- 
22. In Anschluss an diese Darlegung ergeben sich unmittelbar einige Winke in Bezug auf die sachgemässe Prüfung fertiger Mikroskope.

Nach den früher bekannten Thatsachen durfte es als berechtigt erseheinen, den Werth eines Objectivs nach der Kleinheit des letzten Details, welches mit demselben wahnehmbar ist, zu bemessen und daraufhin die Auflösung schwieriger Probeobjecte der bekamnten Art vorzugsweise als Merkmale der Leistungsfähigkeit zu betrachten. Denn wemn auch immer zuzugeben war, dass die besondere Art des Details in diesen Testobjecten und die dabei meist benutzte Beleuchtungsweise nicht zu den gewöhnlichen Vorkommuissen gehören, so schien es doch wenigstens nicht zweifelhaft, dass der Erfolg unter diesen besondern Umstänlen von den nämlichen Eigenschaften der Construction, wie die Leistung im normalen Gebrauch, getragen sein werle. Dies muss nun aber auf Grund der vorstehenden Ausführungen direct abgelehnt werden. Eine Prüfung nämlich, welche auf Ermittelung der äussersten Grenze des Unterscheidungsvermögens, sei er an der Nobert'schen Testiplatte, sei es an Diatomeen oder dergl. ausgeht, füht kraft der physikalischen Bedingungen für diesen Zweck einen ganz exceptionelleu Strahlengang im Mikroskop herbei, wie er bei keiner anderen Art von Beobachtungen jemals wiederkehrt. Denn ein Detail liegt nur dann der Unterscheidungsgrenze nahe, wenn es so fein ist und eine so starke Zerlegung des Lichts durch Beugung bewirkt, dass auch unter den günstigsten Umständen nur der erste abgelenkte

kroskopsysteme sind sämmtlich nach den hier bezeichneten Grundsätzen berechnet und ausgeführt. Dio Trockenobjective haben auch in den stärksten Nummern nur 105-110 Oeffnungswinkel und können demnach nicht den Anspruch machen, in der Auflösung von Diatomeen u. dergl, mit allen andern concurriren zu wollen. Bei den Immersionsobjectiven ist das Maass der freien Oeffnung auf ca. $100^{\circ}$ in Wasser, $d$. h. etwas mehr, als $180^{\circ}$ in Luft entsprechen würde, gestellt worden, weil sich diescs noch ohne elnstliche Nachtheile erreichen lässt. Ioh für meine Person bin zwar iülserzeugt, dass auch das Immersionssystem für die normalen Bedürfnisse des wissenschaftlichen Studiums nicht das Geringste an Werth verlieren, in manchen neben. sächlichen Punkten aber erheblich gewinnen würde, wenn man die Construction anf einen geringeren Oeffnungswinkel einrichten wollte; im Hinblick aber auf den allgemein verbreiteten Maasstab der Werthschätzung kam man einem praktischen Optiker nicht zumuthen, sich um Leistangen zu bemühen, welche' ziemlich sicher wären, in den zweiten Rang gestellt zu werden. 
Lichtbüschel noch eben gleichzeitig mit dem directen Strahlenkegel in das Objectiv eintreten kann. Dann ist aber, wenn es im Bilde sichtbar wird, eo ipso nur die äusserste Raudzone des Objectivs bei der Abbildung thätig: der möglichst. schief einfallende Strahlenkegel, den der Spiegel z. B. liefert, streift den Rand der freien Oeffnung auf der einen Seite und der einzige zur Wirkung gelangende Beugungsbüschel streift ihn an der gegenüberliegenden Seite - wie man durch Beobachtung der Spuren beider in der obern Focalebene des Objectivs direct constatiren kann. Theorje und Erfahrung lehren aber, dass jedes nicht total verfehlte Objectiv, wie unvollkommen es auch im Punkte der sphürischen Aberration sein mag, wenn seine Linsen nur leidlich centrirt sind, inmer für eine einzelne Zone, etwa für den äussersten Rand, aberrationsfrei erhalten werden kann; dauernd - wenn es bei der Verfertigung auf ein derartiges 'Testobject ausprobirt wurde, und während des Gebrauchs - wenn es eine sogen. Correctionsfassung besitzt; welche Vorrichtung denn in der That auch, nach meinen Erfuhrungen, sehr viel öfter zu diesem als zu ihrem ostensibeln Zweck dienen muss.

Der Nachweis, dass ein Linsensystem eine sehr feine Streifung auf einem Diatomeenskelett oder auf der Nobert'schen Platte auflösen kann, besagt also genau genommen nichts weiter, als dass sein Oeffunngswinkel dem berechenbaren Beugungswinkel der betreffenden Liniendistanz entspricht und dass es nicht so schlecht construirt ist, als dass eine ausreichende Correction der Randzone unmöglich wäre. Welche Bedingungen für die correcte Verschmelzung der partiellen Bilder ein solches Objectiv in dem sehr viel ungünstigeren Falle der gewöhnlichen Beobachtung, wo fast immer ganz verschiedene Zonen der freien Oeffnung gleichzeitig thätig sind, darbieten werde, dafür giebt eine Prüfung dieser Art gar keinen Anhalt. Das Resultat derselben kann also nicht einmal den Anspruch machen, auch nur das Unterscheidungs vermögen nach seinen allgemeingiltigen Momenten zu characterisiren; es giebt allein die Unterscheidungs grenze und constatirt damit ein Factum, das zwar an sich einen Werth haben mag, wegen der singulären Umstände aber mit der Höhe der Leistung im Allgemeinen keinen Zusammenhang hat.

Nicht viel höher kanu die Prüfung des Auflösungsvermögens für gerades Licht angeschlagen werden. In der Nähe der dieser Beleuchtungsform entsprechenden Unterscheidungsgrenze passirt das 
directe Licht den centralen und alles abgebeugte Licht den peripherischen Theil der freien Oeffnung. Abgesehen davon, dass mit Hilfe einer Correctionsfassung ein etwaiger Aberrationsrest immer in die unthätig bleibende mittlere \%one des Objectivs geschoben werden kann, hängt auch in diesem Falle das Factum der Auflösung wesentlich nur von der Wirkung der Randzone ab, weil alsdann stets mehrere - mindestens zwoi einander gegenüberliegende - Beugungsbiischel in der Raudzone liegen und diese auch bei mangelhaftem Zusammenwirken mit den ungebeugten Strahlen das Detail hervorrufen.

23. Vom Gesichtspunkte der hier aufgestellten Theorie ergiebt sich hingegen eine andere Methode, welche unter Benutzung der gewöhnlichen Testobjecte unmittelbar die für den normalen Gebrauch des Mikroskops maasgebenden Momente ans Licht zu bringen erlaubt. Wenn es darauf ankommt, die Bedingungen für das correcte Zusammenwirken von Strahlenbüscheln, welche die verschiedensten Theile der freien Oeffnung passiren, auf eine recht empfindliche Probe zu stellen, so gibt es in der That keine bessern Hilfsmittel, als die Natur in manchen Diatomeenskeletten und in einigen Schmetterlingsschuppen darbietet; nur dass allerdings nicht die. Thatsache der "erfolgenden Autlïsung an sich, sondern die nähere Beschaffenheit des entstehenden Gesammtbildes in Betracht gezogen werden muss. Wählt man nämlich ein Probeobject von solcher Feinheit des Details aus, dass ein zu prifendes Objectiv dasselbe schon bei rein centraler Beleuchtung eben sichtbar macht, dass also bei schiefer Beleuchtung nicht die geringste Schwierigkeit bleibt, so kann damit ohne alle weiteren Hilfsmittel der empfindliche Strahlengang im Mikroskop herbeigeführt werden, dessen Herstellung die unter (10) erwähnte Prüfungsmethode durch Beleuchtung des künstlichen Probeobjects mit $z$ w e i getrennten Lichtbüscheln bewirkt. Die Ablenkung der ersten Beugungsbiuschel gewinnt in diesem Falle ein solches Verhältniss zum Oeffnungswinkel des Objectivs, dass - wie die Theorie und die directe Beobachtung der Lichtspuren zeigt - bei zw ei bestimmten Spiegelstellungen Theile aller Zonen der freien Oeffnung, jede durch einzelne Streifen vertieten, wirksam werden, und zwar unter Umständen, welche das Her- . vortreten der Correctionsmängel besonders begünstigen. Die eine erhält man, wenn man den Spiegel senkrecht zu einem Streifensystem der angenommenen Art so weit aus der Axe bewegt, dass 
beiläufig der eine Rand in diese trifft. Alsdann erscheint die Spur des direct eintretenden Lichtkegels im Oeffnungsbild über dem Objectiv excentrisch dicht an der Mitte der freien Oeffnung, die Spur eines Beugungsbüschels aber auf der gegenüberliegenden Seite in der Randzone. Die zweite Stellung ist die der möglichst schiefen Beleuchtung, die das Objectiv ohne merkliche Verdunkelung des Sehfeldes erlaubt; wenn diese herbeigeführt wird, vertauschen die beiden Spuren einfach ihre vorher betrachteten Stellungen. In beiden Fällen hat man, wern nur e in Streifensystem vorliegt, zur Abbildung zwei isolirte Lichtbüschel wirksam, welche einen Theil der centralen und einen Theil der peripherischen Zone der freien Oeffnung, beide anf entgegengesetzten Seiten von der Axe, gleichzeitig in Thätigkeit bringen. Enthält das Präparat mehrere gleichartige Streifungen, so treten zwar noch andere Beugungsbüschel theilweise in das Objectiv ein; doch wird hierdurch an den vorher betrachteten Verhältnissen nichts Wesentliches geändert.

Es kann natürlich nicht der Zweck einer solchen Probe sein, die Abbildungsfehler eines Objectivs im Einzelnen nachzuweisen, so wie die unter (10) erwähnte Methode es erlaubt; es soll sich dabei vieimehr darum handeln, an einem die normalen Bedingungen des mikroskopischen Sehens repräsentirenden Beispiel die thatsächliche Leistungsfühigkeit eines Systems im Ganzen zu erproben. Der praktisch wichtigste Factor derselben ergiebt sich nun sofort, wenn man auf die Verschmelzung der partiellen Bilder, die ein und demselben Theile des Präparats angehören, achtet. Man hat das Contourenbild des Objects, wie es der directe Lichtkegel liefert und zugleich ein Structurbild, welches aus der Interferenz der Beugungsbüschel entspringt. Bei einem correcten Objectiv soll nicht nur jedes für sich vollkommen scharf hervortreten, sondern es sollen zugleich beide ohne Niveaudifferenz und ohne seitliche Verschiebung zusammenfallen, d. h. bei ein und derselben Einstellung deutlich sichtbar sein, wenn im Object Structur und Begrenzung ein und demselben Niveau angehören. Genügt ein Linsensystem dieser, mit ein paar Drehungen der Einstellungsschraube auszuführenden Probe wenigstens in der Mitte des Gesichtsfeldes, so kann man sicher sein, dass es von jedem beliebigen Object und bei jeder Art von Beleuchtung stets richtige Bilder liefern wird: Bemerkt man dagegen, dass, wenn auf die Contouren eingestellt ist, das Detail über oder unter dem Object zu schweben oder seitlich über die Contour hin- 
wegzufliessen scheint, so bekundet ein solcher Befund eine Construction, die Keine Garantie dafür gewährt, an beliebigen Präparaten die zusammengehörigen Merkmale auch als zusammengehörig kenntlich gemacht $\mathrm{zu}$ sehen, wie hoch auch das Aufösungsvermögen des Systems bei der gewöhnlichen Prüfungsmethode sich stellen möge. - Uebrigens kann das Urtheil noch weitere Anhaltepunkte gewinnen, wenn man sich nicht ausschliesslich auf die beiden vorher nambaft gemachten Beleuchtungsformen beschränlrt, sondern auch andere Spiegelstellungen auf ihre Effecte prüt, dabei aber immer auf die charakteristischen Merkmale fur die Verschmelzung der partiellen Bilder achtet. - Dass man bei all diesen Versuchen den wirksamen Strahlengang durch directe Beobachtung des Oeffnungsbildes zu controliren hat, bedarf kaum einer ausdrücklichen Erwähnung.

Bei jedem Objectiv von grossem Oeffnungswinkel wird man $\mathrm{Ab}$ weichungen der angedeuteten Art nach dem Rande des Sehfeldes hin wahrnehmen, wenn nicht etwa der Gesichtswinkel des Oculars ungewöhnlich klein ist. Sie entspringen meist nicht aus Aberrationen, sondern aus Differenzen der Vergrösserung, die bei der besten Construction unvermeidlich sind. Der Grad ihres Hervortretens bemisst die Vollkommenheit der Abbildung ausser der Axe.

Was ausserdem zur Vervollständigung des Urtheils über die Güte eines Linsensystems gehört, giebt ein Blick auf die Farbensäume, welche die Contouren eines Probeobjects in der Mitte und am Rande des Sehfeldes zeigen. Dabei ist zu beachten, dass Abweichungen dieser Art, weil sie vorwiegend am Contourenbild haften, beim gewöhnlichen Gebrauch des Mikroskops, der fast immer auf gerade Beleuchtung angewiesen ist, nur in dem Maasse eine praktische Bedeutung haben, als sie bei centraler Spiegelstellung hervortreten.

Was nun die Probeobjecte anlangt, welche sich zum Gebrauch in der angegebenen Richtung eignen, so müssen sie namentlich zweien Anforderungen genügen. Erstens müssen sie so dünn und eben sein, dass man Grenzen und Structurdetail als in dem nämlichen Niveau liegend ansehen kann; zweitens müssen die abgebeugten Strahlen eine grosse Intensität haben, damit der von ihnen herrührende Effect neben dem des directen Lichtkegels gehörig zur Geltung kommen kann. Der letztern Rücksicht wegen eignen sich also nur trocken liegeude Objecte mit kräftiger, gut markirter 
Zeichnung, welche - wie man im Oeffnungsbild beobachten kann - stets sehr lichtstarke Bengungsphänomene geben, weil eben nur die Interferenz intensiver Strahlen starke Contraste von Hell und Dunkel im mikroskopischen Bild hervorrufen kann.

Für die schwächeren und mittleren Objective hat man in den Insectenschuppen und den gröberen Diatomeen, welche die mikrographischen Handbücher aufführen, eine himreichende Zahl passender Objecte zur Verfügung; für die starken Objective dagegen wird die Auswahl sehr beschränkt durch die Rücksicht auf die erstgenannte Anforderung ${ }^{1}$ ). Dem Oeffnungswinkel der Immersionssysteme entspricht, der Feinheit des Details nach, Pleur. ang. durchschnittlich am besten; es ist auch in der that noch für die schärfsten Nummern sehr gut brauchbar, wenn man von recht zarten Exemplaren Bruchstü cke mit scharfen Bruchrändern verwendet und das Augenmerk nur auf die Beschaffenheit des Bildes dicht an einer solchen Randstelle richtet; die natürlichen Ränder bieten ebensowenig wie die Linien der Mittelrippe Garantie für Gleichheit des Niveaus. Zur Prüfung der stärlkeren Trockensysteme können die gröberen Exemplare desselben Objects, gleichfalls in Bruchstücken, noch benutzt werden, wiewohl die Zeichnung für einen Oeffnungswinkel von $100 \mathrm{Grad}$ schon etwas zu fein ist. Ausserdem kann man zerbrochene Exemplare der feineren Schuppen von Hipparchia Jan, deren Querstreifen für 80-900 Oeffnungswinkel passen, auch für höhere Beträge desselben ohne Nachtheil verwenden.

Meiner Erfahrung zufolge führt eine Untersuchung in dieser Art nach einiger Uebung zu einem sehr sichern Urtheil über die Vollkommenheit eines Linsensystems; wenigstens wird die Höhe des optischen Vermögens in seinen von der Grösse des Oeffnungswinkels unabhängigen Functionen sehr viel zutreffender eruirt als durch die getrennten Proben auf Begrenzungs- und Auflösungsvermögen jemals geschehen kann.

Was aber diese Methode nicht ergiebt - die absolute Grenze des physischen Unterscheidungsvermögens - kann durch blosse Messung des Oeffnungswinkels ebenso gut wie durch directe Erpro-

1) Die Nobert'sche Testplatte ist natürlich für diese Art der Prüfung ungeeiguet, weil sio überhaupt kein Absorptions- oder, Contourenbild, sondern ein reines Beugungsbild liofert und desshalb das wiohtigste Moment für die Beurtheilung der Wirkung bei ihr hinwegfallt. 
bung an Testobjecten erhalten werden. - Wegen des Verfahrens, durch welches, der Oeffnungswinkel in jeder nur wünschenswerthen Genauigkeit zu erlangen ist, verweise ich auf die Jenaische Zeitschr.

24. Zum Schluss sei noch einiger allgemeiner Folgerungen in Bezug auf die Construction der Mikroskope gedacht, welche die dargelegten Thatsachen und Theorien aach sich ziehen.

Den gegebenen Nachweisen zufolge hängt die Leistungsfähigkeit des Mikroskops von $z$ wei Factoren $-a b$, welche in ganz verschiedenen Elementen der Construction wurzeln. Der eine ist die geometrische Vollkommenheit des Strahlengangs. Sie bestimmt durch die Grösse der Zerstreuungskreise in der Bildfläche die Maasse des kleinsten Details, welches rein geometrisch genommen im Bilde Ausdruck finden kann. Der zweite Factor dagegen ist die Fähigkeit des optischen Apparats, die physische Bedingung zu erfüllen, an welche die Wiedergabe solchen Details jedenfalls geknüpft ist, nämlich die Integration des durch Beugang zerlegten Lichtes, ohne welche das Bild inhaltleer bleibt. Wie aus geometrischen Gründen ein Detail nicht abgebildet wird, wenn seine Maasse unterhalb der Grösse bleiben, welche dem Durchmesser der Zerstreungskreise, auf die linearen Dimensionen des Objects reducirt, entspricht, so wird es aus physikalischen Gründen nicht abgebildet, wenn die Winkelausbreitung des Beugungsphänomens durch ihre Grösse eine Vereinigung von mindestens zwei- Beugungsbüscheln unmöglich macht. Dem früher Gesagten zufolge wurzeln nun zwar die Bedingungen für beide Functionen in demselben Bestandtheil des optischen Systems, nämlich allein im Objectiv; aber sie wurzeln in ganz verschiedenen Elementen seiner Construction. Die dioptrische Unterscheidungsgrenze, welche die unvermeidlichen Mängel der Strahlenvereinigung herbeiführen, findet ihr Maass in der förderlichen Vergrösserung des Objectivs und ist, wie früher angegeben, für jeden bestimmten Grad der technischen Vollendung der Construction der Brennweite des Objectivs umgekehrt proportional; die physikalische Unterscheidungsgrenze dagegen hängt allein vom Oeffnungswinkel $a b$ und ist dem Sinus seines halben Betrages proportional. Nun sind aber beide Functionen auf ein und denselben Zweck gerichtet, nämlich auf das Sichtbarmachen des räumlich Kleinen, und sind für diesen $Z$ weck beide gleich uneutbehrlich. Daraus folgt denn, dass eine rationelle Construction des Mikroskops darauf Bedacht nehmen muss, sie in der Weise ins Gleichgewicht zu setzen, dass die Grenzen 
beider wenigstens annähernd zusammentreffen. Denn es ist offenbar ebenso nutzlos, den physischen Bedingungen der Abbildung in weiterem Umfang genügt zu haben, als die erreichbare Vergrösserung zu verwerthen erlaubt, wie es nutzlos ist, die Vergrösserungskraft des Mikroskops höher zu steigern, als die physische Capacität der Objective nöthig macht. In ersten Fall, wenn der Oeffnungswinkel zu gross ist für die förderliche Vergrösserung, die der Brennweite entspricht, gewinnt das Objectiv ein latentes Auflösungsvermögen, welches keinem menschlichen Ange zu Gute kommt; im andern Fall, wenn die Stärke des Objectivs die dioptrische Unterscheidungsgrenze weiter hinausrückt, als das dem Oeffnungswinkel zugängliche Detail nöthig macht, entsteht eine leere Vergrösserung, d. h. eine solche, die im Bilde nichts vorfindet, was ihrer bedürfte.

\$5. Die Consequenzen dieser Erwägung führen zu gewissen Maximen für die richtige Anpassung zwischen Brennweite und Deffnungswinkel bei den Objectiven, welche den Gewohnheiten der bisherigen Praxis in mehreren Punkten widersprechen. Hier mag Dasjenige Platz finden, was mir von allgemeinerem Interesse zu sein scheint, ẉeil es die Tragweite der mikroskopischen Beobachtung im Ganzen ins Licht setzt und die Ergäuzung der unter (9) gegebenen Darlegungen liefert.

Wenn für das Trockensystem die Theorie eine Beschränkung des Oeffnungswinkels auf ca. $110^{\circ}$ unbedingt fordert, so kann man leicht ausrechnen, welches die feinste Structur ist, die diesem Oeffnungswinkel zugänglich bleibt; und es ergiebt sich darauf hin, dass wenigstens für ein rationell construirtes Objectiv dieser Art, bei welchem bicht auf Kosten der wirklichen Vollkommenheit die Auflösung einseitig gesteigert ist, kein Detail in Frage kommen kann, das ein geïbtes Auge nicht schon bei einer guten 4-500fachen Vergrösserung zu erkennen vermöchte. Bei den Ansprüchen, welche nach dem dermaligen Stand der optischen Technik an die relative Vollkommenheit der Constructionen gestellt werden dürfen, muss aber diese Höhe der Vergrösserung - auch wenn man es mit dem Attribut vgut« etwas strenger nimmt, als ofters geschieht - schon mit einer Brennweite von ca. $3 \mathrm{Mm}$. ( $1 / 8$ engl. Zoll) erreicht sein. Beirn Immersionssystem rückt, selbst wenn der Oeffnungswinkel auf das höchste technisch erreirhbare Maass gebracht wird, die physische Unterscheidungsgrenze doch nicht so weit zurück, dass ihr nicht eine correcte 7-800fache Vergrösserung vollkommen gewachsen bliebe, 
und diese muss hier bei guter Construction mit ca. $2 \mathrm{Mm}$. (1/12 Zoll) Brennweite sicher zur Verfügung stehen. Nun wird man zwar zugeben, dass eine weitere möglichst correcte Vergrösserung über das unerlässliche Minimum hinaus, obwohl sie die Wahrnehmung nicht mit neuen 'Thatsachen bereichern kann, doch der Leichtigkeit und Sicherheit der Beobachtung sehr zu Statten kommen mag. Indess wird man diese Bedeutung der leeren Vergrösserung schwerlich weit über die bezeichneten Grenzen hinaus anerkennen dürfen; und ich komme daher zu dem Schlusse, dass der wissenschaftliche Werth von Objectiveu, deren Brennweite beim Trockensystem unter $2 \mathrm{Mm}$., beim Immersionssystem unter $1 \mathrm{Mm}$. erheblich herabgeht, durchaus problematisch ist.

Die eigentliche Capacität des Mikroskops im strengeren Sinne aber muss ich - so lange nicht Momente geltend gemacht werden, die ganz ausserhalb der Tragweite der aufgestellten Theorie liegen - schon bei der oben bezeichneten fruheren Grenze als vollständig erschöpft ansehen; und im Besondern muss ich die Ansicht vertreten: dass mit keinem Mikroskop irgend etwas in der Beschaffenheit der Objecte wirklich Begrundetes jemals gesehen worden ist and gesehen werden kann, was ein normales Auge nicht auch schon mit einer scharfen 800 fachen Inmersionsvergrösserung sicher zu erkennen vermöchte. Was in neuerer Zeit, zumal aus England, über ganz ausserordentliche Leistungen ungewöhnlich starker Objective (bis $1 / 80$ engl. Zoll Brennweite) berichtet worden ist, ist nicht darnach angethan, mich in diesem Urtheil irre zu machen. Denn die Ueberlegenheit solcher Linsensysteme soll an Objecten constatirt sein, auf welche die unmittelbaren Ergebnisse meiner Experimente bedingungslọ Anwendung finden; und sie soll unter Vergrösserungen zu Tage treten, deren Höhe Jeder als völlig illusorisch erkennt, der sich von den optischen Bedingungen einer solchen Leistung einige Rechenschaft geben kann. 